\title{
Densely Functionalized 2-Methylideneazetidines: Evaluation as Antibacterials
}

\author{
Giovanni Petrillo ${ }^{1, *}{ }^{(}$, Cinzia Tavani $^{1}{ }^{1}$, Lara Bianchi $^{1}$, Alice Benzi ${ }^{1}$, Maria Maddalena Cavalluzzi ${ }^{2}$,

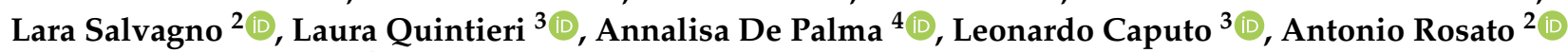 \\ and Giovanni Lentini ${ }^{2}$ (D)
}

1 Department of Chemistry and Industrial Chemistry, University of Genoa, Via Dodecaneso 31, I-16146 Genoa, Italy; cinzia.tavani@unige.it (C.T.); lara.bianchi@unige.it (L.B.); alice.benzi@edu.unige.it (A.B.)

2 Department of Pharmacy-Pharmaceutical Sciences, University of Bari Aldo Moro, Via E. Orabona n. 4, 70126 Bari, Italy; mariamaddalena.cavalluzzi@uniba.it (M.M.C.); lara.salvagno89@gmail.com (L.S.); antonio.rosato@uniba.it (A.R.); giovanni.lentini@uniba.it (G.L.)

3 Institute of Sciences of Food Production (CNR-ISPA) National Council of Research, Via G. Amendola, 122/O, 70126 Bari, Italy; laura.quintieri@ispa.cnr.it (L.Q.); leonardo.caputo@ispa.cnr.it (L.C.)

4 Department of Biosciences, Biotechnologies, and Biopharmaceutics, University of Bari Aldo Moro, Via E. Orabona 4, 70126 Bari, Italy; annalisa.depalma@uniba.it

* Correspondence: giovanni.petrillo@unige.it; Tel.: +39-010-353-6103

check for updates

Citation: Petrillo, G.; Tavani, C.; Bianchi, L.; Benzi, A.; Cavalluzzi, M.M.; Salvagno, L.; Quintieri, L.; De Palma, A.; Caputo, L.; Rosato, A.; et al. Densely Functionalized 2-Methylideneazetidines: Evaluation as Antibacterials. Molecules 2021, 26, 3891. https://doi.org/10.3390/ molecules26133891

Academic Editor: Athina Geronikaki

Received: 18 May 2021

Accepted: 22 June 2021

Published: 25 June 2021

Publisher's Note: MDPI stays neutral with regard to jurisdictional claims in published maps and institutional affiliations.

Copyright: (C) 2021 by the authors. Licensee MDPI, Basel, Switzerland. This article is an open access article distributed under the terms and conditions of the Creative Commons Attribution (CC BY) license (https:/ / creativecommons.org/licenses/by/ $4.0 /)$.

\begin{abstract}
Twenty-two novel, variously substituted nitroazetidines were designed as both sulfonamide and urethane vinylogs possibly endowed with antimicrobial activity. The compounds under study were obtained following a general procedure recently developed, starting from 4nitropentadienoates deriving from a common $\beta$-nitrothiophenic precursor. While being devoid of any activity against fungi and Gram-negative bacteria, most of the title compounds performed as potent antibacterial agents on Gram-positive bacteria (E. faecalis and three strains of S. aureus), with the most potent congener being the 1-(4-chlorobenzyl)-3-nitro-4-( $p$-tolyl)azetidine 22, which displayed potency close to that of norfloxacin, the reference antibiotic (minimum inhibitory concentration values 4 and $1-2 \mu \mathrm{g} / \mathrm{mL}$, respectively). Since 22 combines a relatively efficient activity against Gram-positive bacteria and a cytotoxicity on eucharyotic cells only at 4-times higher concentrations (inhibiting concentration on $50 \%$ of the cultured eukaryotic cells: $36 \pm 10 \mu \mathrm{M}$, MIC: $8.6 \mu \mathrm{M}$ ), it may be considered as a promising hit compound for the development of a new series of antibacterials selectively active on Gram-positive pathogens. The relatively concise synthetic route described herein, based on widely available starting materials, could feed further structure-activity relationship studies, thus allowing for the fine investigation and optimization of the toxico-pharmacological profile.
\end{abstract}

Keywords: antibiotics; drug resistance; gut microbiota; vinylogy

\section{Introduction}

Eighty years ago, the modern era of antimicrobial therapy started. Since then, hundreds of antibacterials have been successfully used as clinically valuable agents to fight one of the main threats to human health, substantially contributing to the prolongation of life expectancy in the past century. Unfortunately, the widespread use (and misuse) of the 'miracle drugs' [1] has caused the emergence of antibacterial-resistant microorganisms, most of which display pathogenic potential. In particular, six multidrug-resistant pathogens (MDR: Enterococcus faecium, Staphylococcus aureus, Klebsiella pneumoniae, Acinetobacter baumannii, Pseudomonas aeruginosa, and Enterobacter spp.) were named ESKAPE pathogens due to their ability to 'escape' the biocidal action of antimicrobials [2]; such pathogens are responsible for the majority of nosocomial infections associated with higher rates of morbidity and mortality. The World Health Organization (WHO) has also recently classified ESKAPE pathogens in a list of 12 pathogens grouped as critical, high and medium 
priority, according to the urgency for new antibiotics [3]. The critical-priority list includes carbapenem resistant A. baumannii, P. aeruginosa, K. pneumoniae, and Enterobacter spp.; whereas vancomycin-resistant $E$. faecium (VRE) and methicillin and vancomycin-resistant S. aureus (MRSA and VRSA) are listed in the high-priority group. Besides the need to improve the surveillance protocols of antimicrobial resistance, it is urgent to find alternative strategies to cure infections, especially those caused by ESKAPE pathogens [4].

The compelling need for new efficient drugs to support antimicrobial chemotherapy is demonstrated by the ever-increasing number of research groups focusing on this issue, mirrored, in turn, by the increase displayed by the number of relevant publications in the last five lustra (Figure 1).

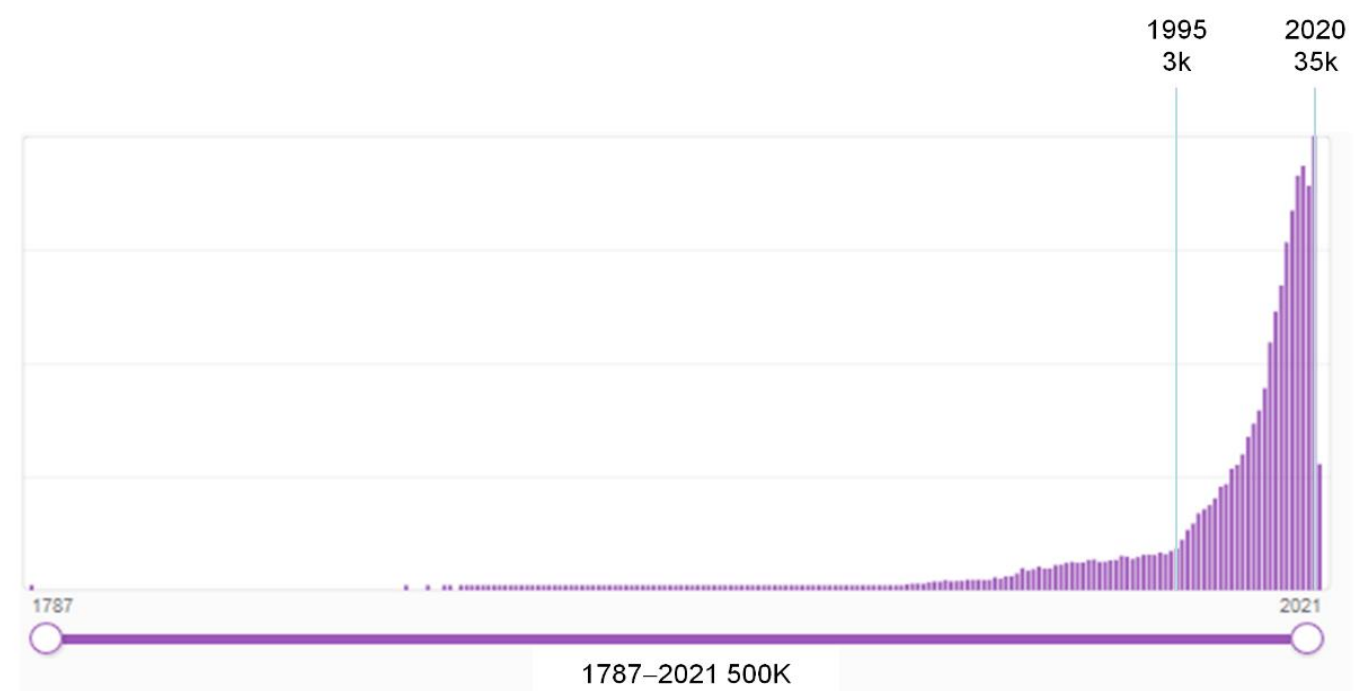

Figure 1. Number of answers per year from SciFinder ${ }^{\mathrm{n}}$ to the query 'antibacterials' [5].

Despite the effort, success in the development of antibacterial agents has been limited since several aspects (i.e., potency, pharmacokinetics and safety) have to be optimized when trying to rationally design new antibacterials, while the corresponding parameters are often contrasting [6]. The phenotypic screening of small libraries, i.e., whole bacteria treated with small libraries of molecules, seems to be a relatively less demanding approach to finding hit compounds [7].

When considering the most successful, commonly used classes of antibacterials, we noticed the frequent presence of some structural elements such as the azetidine core and sulphonamide, carbamate, and nitro groups (Figure 2). Thus, we speculated that gathering all of the above motifs in the same structure would confer antibacterial activity to the so-designed small molecule congeneric series (5-26). The general structure reported in Figure 2 could be intended as the result of a scaffold-hopping strategy [8] applied to both sulfonamides and $\beta$-lactams. For the vinylogy principle [9], the methylsulfonyl group (green) should behave as a sulphonamide and the methoxycarbonyl group (blue) should be roughly endowed with the same electron density of a carbamate. As a matter of fact, a series of azetidine-containing compounds showed antibacterial, antifungal and antitubercular activities in vitro depending on the substituent on the 4-aryl group, according to the sequence: $\mathrm{NO}_{2}>\mathrm{Cl}>\mathrm{Br}>\mathrm{OH}>>\mathrm{OCH}_{3}>\mathrm{CH}_{3}$ [10]. Conversely, among six azetidines bearing different phenyl/heterocyclic moieties, the one bearing the indole moiety displayed the highest antibacterial activity, probably due the resemblance with the $\beta$-lactam ring [11].

Considering that the introduction of a nitro group generally improves the antibacterial activity, we felt encouraged to prepare the nitroazetidines 5-26 and to test them as antimicrobials. 


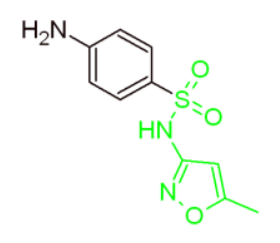

sulfamethoxazole (1) sulfonamide

folate synthesis inhibitor

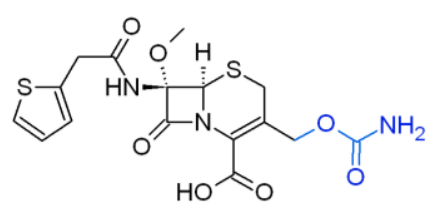

cefoxitin (3)

cephalosporin

cell wall synthesis inhibitor

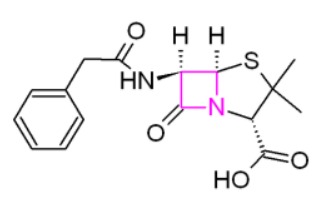

penicillin G (2)

$\beta$-lactam

peptidoglycan cross-linking inhibitor

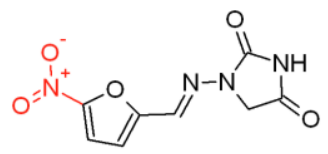

nitrofurantoin (4)

nitrofuran

mRNA translation inhibitor

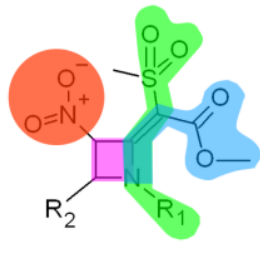

5-26

Figure 2. Structures of commonly used antibacterial agents characterized by a sulfonamide group (1), an azetidine core (2), a carbamate group (3) or a nitro substituent (4), and the general structure for the designed compounds 5-26. Antibacterial classification and mechanism of action were included.

\section{Results and Discussion}

The title compounds were obtained according to the procedure reported in Scheme 1 [12], which, starting from nitropentadienoates in turn derived from a common nitrothiophenic precursor (Scheme 1), resulted in general efficiency and applicability. Briefly, the ringopening of the thiophenic precursor 27 by pyrrolidine produces the nitroenamine 28 , from which the aryl pentadienoates $\mathbf{2 9}$ and $\mathbf{3 0}$ can be conveniently obtained. Treating $\mathbf{3 0}$ with primary amines, the corresponding aza-Michael adducts are generated, which cyclize to the nitroazetidines of interest.

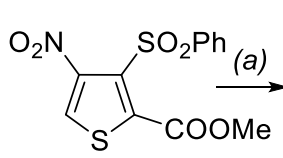

27<smiles>COC(=O)C(C(=O)OC)=C(/C=N/N1CCCC1)[N+](=O)[O-]</smiles>

28

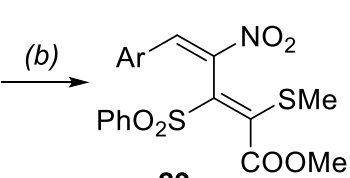

29

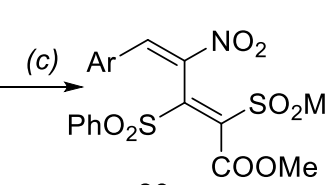

30

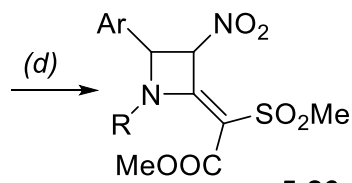

$5-26$

Scheme 1. Synthesis of azetidines 5-26 starting from the common precursor 27. (a): pyrrolidine, $\mathrm{AgNO}_{3}, \mathrm{EtOH}, \mathrm{then} \mathrm{MeI}$; (b): ArMgX, THF, then acidic quenching; (c): $m$-CPBA, DCM; (d): $\mathrm{RNH}_{2}, \mathrm{DCM}$.

The title compounds were tested as antimicrobials against selected pathogens belonging to the ATCC collection; they included Gram-positive bacteria Staphylococcus aureus (ATCC 29213, 6538 P, and 6538), and Enterococcus faecalis (29212), Gram-negative bacteria Pseudomonas aeruginosa (ATCC 27853), Escherichia coli (ATCC 25922), and Klebsiella pneumoniae (ATCC 13883), and fungi Candida albicans (ATCC 10231), Candida albicans (ATCC 90028), Candida glabrata (ATCC 15126), Candida tropicalis (ATCC 750), Candida kefyr (ATCC 204093), Candida krusei (ATCC 6258). Clinical isolates (Candida albicans A18, Candida albicans 810, Candida krusei 31A29, Candida parapsilosis 11A13, Candida parapsilosis 1A1) were also included. The activity was measured as the minimum inhibitory concentration (MIC) that is the concentration $(\mu \mathrm{g} / \mathrm{mL}, \gamma)$ that prevents any visible bacterial growth. All the compounds tested were inactive against both Gram-negative bacteria and fungi (MIC $>512 \gamma$ ) but displayed significant activity against Gram-positive bacteria (Table 1). These results were in accordance with the spectrum of activity of $\beta$-lactams antibiotics; these latter, indeed, were able to induce cell wall destabilization after diffusing through the bacterial cell wall. In contrast to Gram-positive bacteria, Gram-negative ones have an additional lipopolysaccharide layer that decreases antibiotic penetration. 
In general, the most active compounds were the benzyl $N$-derivatives 14-26, with interesting differences based on the number $(n=0-2)$ and position of chlorine atoms on the phenyl ring. In particular, the $o<m<p$ trend of activity was observed for the $N$ chlorobenzyl analogues 16-18 and 20-22, thus indicating the para position as the preferred one for the antibacterial activity. When a second chlorine atom was introduced in the most potent $p$-chloro substituted compounds (18 and $\mathbf{2 2}$ ) to obtain the 3,4-dichlorobenzyl analogues 19 and 23, respectively, the activity was mostly affected in the $p$-tolyl series with compound 23 showing 2-8-fold higher MIC values (i.e., lower activity) against the four bacterial species tested. On the contrary, the absence of a chlorine atom led to a total loss of activity against $E$. faecalis in the $\mathrm{R}^{1}=$ benzyl, $\mathrm{R}^{2}=p$-tolyl azetidine derivatives (14, 20-23), with the MIC value of 14 being $>512 \mu \mathrm{g} / \mathrm{mL}$; no dramatic effects were observed in the 1benzyl-2-thienyl series (15-19). Furthermore, a peculiar behavior was observed in S. aureus ATCC 6538 in both $p$-tolyl and 2-thienyl azetidine series, since a higher activity was reached when the chlorine atom is absent rather than present in the ortho and ortho/meta positions, respectively. In general, among the benzyl analogues 14-23, slightly higher activity on all tested strains was observed when a $p$-tolyl moiety, rather than 2-thienyl, was linked at the 2-position of the azetidine core, regardless of the substituent in the 1-position. The alkyl or cycloalkyl substitution (5-12) was not well tolerated, leading to a loss of potency (MIC $>512 \mu \mathrm{g} / \mathrm{mL}$ ), except for compound 12 showing the same MIC value as 16 against two of the three S. aureus strains (ATCC 29,213 and ATCC 6538P). Unlike the latter strains, 16 was completely inactive against $E$. faecalis. In general, the observed antibacterial activity was not strictly correlated with lipophilicity $(\operatorname{cog} P)$. However, a roughly parabolic trend might be seen, with optimal cLog $p$ values being within the 1-3 range. The highest activity was obtained for compound 22, whose MIC value $(4 \mu \mathrm{g} / \mathrm{mL})$ was only 2 -fold higher than the reference compound norfloxacin against the E. faecalis, an important cause of life-threatening multidrug-resistant bacterial nosocomial infections.

Looking for a possible mechanism behind the observed antibacterial activity, a preliminary study was carried out to see if our compounds might act via bacterial cytoplasmic membrane disruption. Increases in cell envelope permeability after treatment with the nitroazetidines 18, 21 and 22 were analyzed by the crystal violet (CV) assay, through evaluation of the percentage of its uptake after incubation with the antimicrobial agent as previously reported $[13,14]$. The increase in CV uptake was generally associated with the membrane leakage due to a permeability change [13-15]. The use of the antibiotic bacitracin, well known to inhibit cell wall biosynthesis [16], as reference for the assay, confirmed the increase of CV uptake also when the cell wall integrity was compromised. Both S. aureus ATCC 6538 P and E. faecalis ATCC 29,212 strains were sensitive to bacitracin as reported by the BacDive database [17]. As reported in Figure 3, untreated cells registered ca. 51\% uptake. As expected, a significant dose-response effect for bacitracin on CV uptake was registered for both strains $(p<0.05$; ranging from 57.63 to $83.97 \%$, with concentration values from $0.1 \mathrm{mg} / \mathrm{mL}$ to $1 \mathrm{mg} / \mathrm{mL}$ ). A slight but significant increase in CV uptake values (56.34\%, on average) was registered only for S. aureus cells treated with 18 and 21 at concentrations 8 and 10-fold higher than their MIC. No differences were instead registered for E. faecalis regardless of the assayed compounds. Although a slight membrane permeability was registered for 18 and 21, the results exclude that the antimicrobial activity registered against $S$. aureus and $E$. faecalis might be caused by a cell envelope destabilization. 

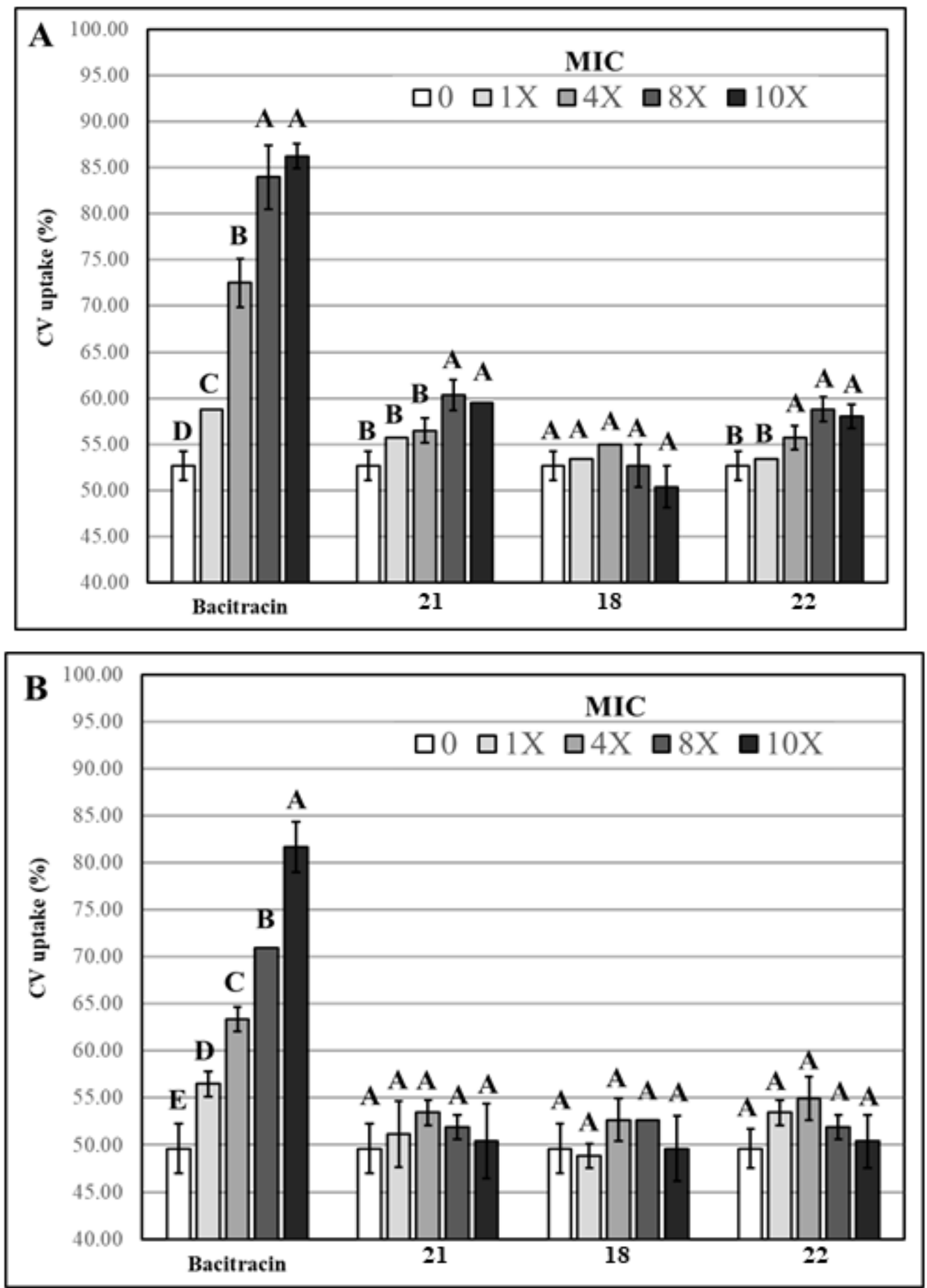

Figure 3. Crystal Violet (CV) uptake assay. The percentage (\%) of CV uptake from S. aureus ATCC 6538P (A) and E. faecalis ATCC 29,212 cells (B), either untreated (0) or treated with bacitracin, 21, 18 and 22 at $1 \mathrm{X}, 4 \mathrm{X}, 8 \mathrm{X}, 10 \mathrm{X}$ MIC for $1 \mathrm{~h}$ at $37^{\circ} \mathrm{C}$. Bars represent the means \pm standard deviation. Different superscript letters A-E indicate average values significantly different $(p<0.05)$ according to post hoc Tukey test.

Although the $\beta$-lactam mechanism of action is due to the covalent interaction with the penicillin-binding proteins (PBPs), involved in peptidoglycan biosynthesis, structural changes ( $N$-alkylation, ring opening and type of substituents at the $\mathrm{C} 3$ and $\mathrm{C} 4$ positions) can determine additional modes of action (modifications of the bacterial cell uptake, membrane permeability or inhibition of fatty acid synthesis) as suggested by Revell et al., 2007 [18]. Recently, azetidines endowed with bactericidal activity against Mycobacterium tuberculosis 
were also synthesized; mode of action and target deconvolution studies suggest that these compounds inhibit mycobacterial growth by interfering with cell envelope biogenesis, specifically late-stage mycolic acid biosynthesis. In addition, these compounds did not give rise to emerging specific resistance in mycobacterial model organisms; the inability to generate resistant mutants against the most active compounds suggests that this series of compounds may elicit pleiotropic activity or have non-specific modes of action, or have non-protein target(s) [19].

Since the structures of nitroazetidines 5-26 include an allegedly electron-poor ylidene $\mathrm{C}=\mathrm{C}$ bond, it might be hypothesized that they may act as covalent modifiers of transpeptidase-like $\beta$-lactams. This would be in agreement with the observed high selectivity towards Gram-positive bacteria. To check this hypothesis, we run computational investigations on the geometrical and electronic properties of compound 22, to be compared with the corresponding ones in penicillin G (2), chosen as the most typical antibacterial $\beta$-lactam. The relationships between $\beta$-lactam stereoelectronic features and the biological activity are well established and are qualitatively reported in the fourth column of Table 2 [20].

The study was run at the DFT $\tilde{\omega}$ B97X-D/6-31G* level (gaseous phase) on one of the two most stable conformations of $\mathbf{2}$, namely the folded one (Figure $4 \mathrm{~A}$ ) [21,22]. The same analysis was performed on the global minimum conformer of 22, obtained through our previously developed conformational analysis procedure (Figure 4B). [23,24].

Most of the properties reported in Table 2 indicate that the azetidine ring in our compounds might hardly be considered as a potential electrophilic reagent like $\beta$-lactams, with the only electrophilicity index $\tilde{\omega}$ winking to that direction. On the contrary, the distribution found in the HOMO density maps (Figure 4C,D) seems to assign a local nucleophilic potential to the ylidene $\mathrm{C}=\mathrm{C}$ bond. What is discussed above seems to indicate that compound 22 should not act as a covalent modifier of transpeptidase even though this conclusion does deserve further theoretical and experimental studies. As an early observation supporting the relatively low electrophilic potential of our compounds, we have observed high stability of azetidine 5-26 under harsh alkaline conditions.

Since aliphatic nitro-derivatives are expected to be potentially toxic to eukaryotic cells [25], the most efficient [26] antibacterial congeners 18, 21 and 22 underwent preliminary acute toxicity profile evaluation through the platform Percepta (ACD/labs 2020.1.0). The software indicated compounds 18, 21 and 22 as agents potentially able to cause hepatic damage (median $\mathrm{LD}_{50}: 120 \mathrm{mg} / \mathrm{Kg}$ for 11 aliphatic nitro-compounds in the training database). Compounds 18, 21 and 22 were predicted as equally moderately hazardous since they share the same $\mathrm{LD}_{50}$ values in each animal model:

mouse (ip): $190 \mathrm{mg} / \mathrm{kg}$

mouse (po): $2300 \mathrm{mg} / \mathrm{kg}$

mouse (iv): $56 \mathrm{mg} / \mathrm{kg}$

mouse (sc): $46 \mathrm{mg} / \mathrm{kg}$

rat (ip): $51 \mathrm{mg} / \mathrm{kg}$

rat (po): $2400 \mathrm{mg} / \mathrm{kg}$.

The most efficient congener 22 was then tested for cytotoxicity on eukaryotic cells (hepatocarcinoma cells, HepG2) [27] and displayed detrimental effects only at 4-times higher concentrations than its MIC (inhibiting concentration on $50 \%$ of the cultured eukaryotic cells: $36 \pm 10 \mu \mathrm{M})$. 
Table 1. Antibacterial activity of nitroazetidines 5-26 and norfloxacin (reference antibacterial agent) ${ }^{a}$.

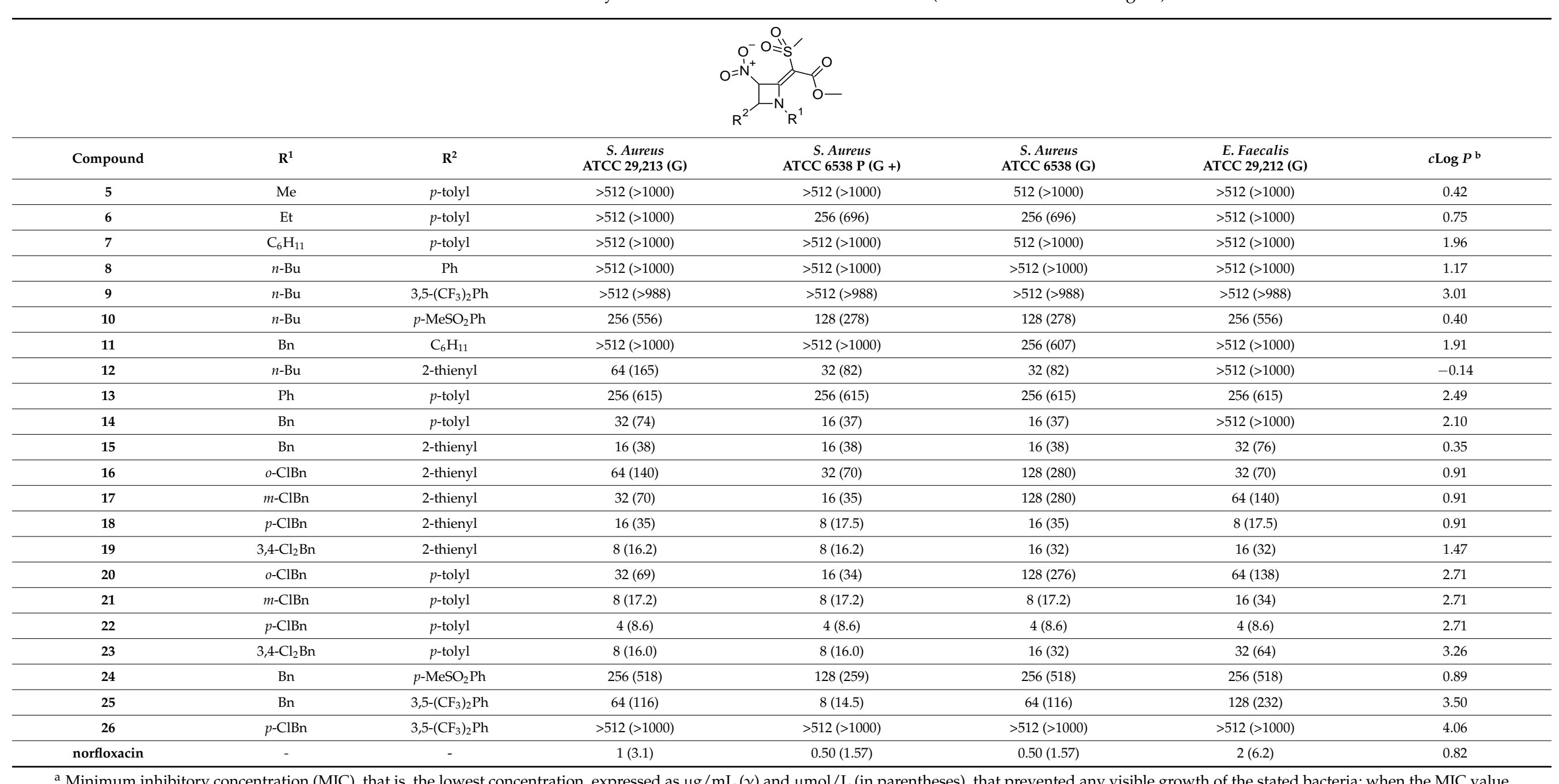

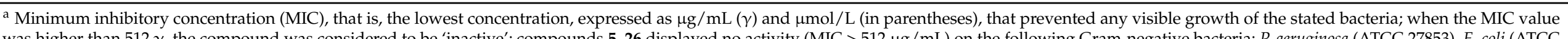

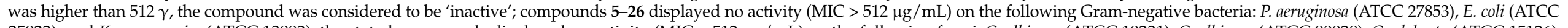

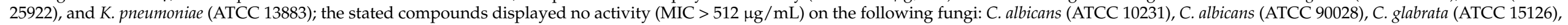

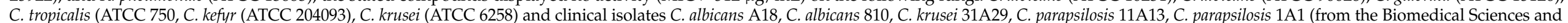

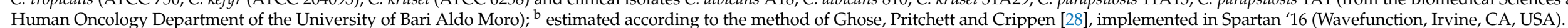


Table 2. Comparison of the Main Stereoelectronic Features of the Azetidine Rings in Penicillin G and 2-Nitroazetidine 22 as Obtained through Quantum-Mechanical Calculations (DFT $\tilde{\omega}$ B97X-D/6-31G*//DFT $\tilde{\omega}$ B97X-D/6-31G*, Gaseous Phase).<smiles>[X]C1C([R])N([R7])C1[R7]</smiles>

\section{Penicillin $\mathrm{G}^{\mathrm{a}}$}

\section{Compd 22 ${ }^{b}$}

Reported qualitative relationship between $\beta$-lactam stereoelectronic features and antibacterial activity [ref1]; $\uparrow:$ improves, $\downarrow$ : lowers

\begin{tabular}{|c|c|c|c|}
\hline$C=X$ bond length $(\AA)$ & 1.20 & 1.37 & short $\uparrow$; long $\downarrow$ \\
\hline C-N bond length $(\AA)$ & 1.40 & 1.35 & long $\uparrow$; short $\downarrow$ \\
\hline $\mathrm{C}=\mathrm{X}$ bond order & & & high $\uparrow$; low $\downarrow$ \\
\hline Löwdin & 2.22 & 1.55 & \\
\hline Mulliken & 1.92 & 1.53 & \\
\hline $\mathrm{C}=\mathrm{X}$ stretching frequency $\left(\mathrm{cm}^{-1}\right)^{\mathrm{c}}$ & 1838 & 1624 & high $\uparrow$; low $\downarrow$ \\
\hline $\begin{array}{l}\text { net atomic charge on } \mathrm{X} \\
\text { (units of electrons) }\end{array}$ & & & less negative $\uparrow$; more negative $\downarrow$ \\
\hline electrostatic & -0.45 & -0.67 & \\
\hline Mulliken & -0.46 & -0.40 & \\
\hline natural & -0.56 & -0.57 & \\
\hline Sum of bond angles at nitrogen $\left(^{\circ}\right)$ & 332 & 359 & $<<360 \uparrow ;<360 \downarrow$ \\
\hline Woodward $h$ value ${ }^{\mathrm{d}}$ & 0.46 & 0.19 & high $\uparrow$; low $\downarrow$ \\
\hline electrophilicity index $(\mathrm{eV})^{\mathrm{e}}$ & 0.90 & 1.05 & high $\uparrow$; low $\downarrow$ \\
\hline
\end{tabular}

${ }^{\mathrm{a}} \mathrm{X}=\mathrm{O} ; \mathrm{R}^{1}, \mathrm{R}^{2}=\mathrm{CH}\left(\mathrm{CO}_{2} \mathrm{H}\right) \mathrm{C}\left(\mathrm{CH}_{3}\right)_{2} \mathrm{~S} ; \mathrm{R}^{3}=\mathrm{BnC}(\mathrm{O}) \mathrm{NH} .{ }^{\mathrm{b}} \mathrm{X}=$ quaternary $\mathrm{C}\left(\mathrm{sp}^{2}\right) ; \mathrm{R}^{1}=p$-ClBn; $\mathrm{R}^{2}=p$-tolyl; $\mathrm{R}^{3}=\mathrm{NO}_{2} .{ }^{\mathrm{c}} \mathrm{DFT}$ EDF2 $/ 6$ $31 G^{*} / /$ DFT $\tilde{\omega} B 97 X-D / 6-31 G^{*}$, gaseous phase; the so-calculated IR frequencies are typically overestimated by $5 \% .{ }^{d}$ distance of the nitrogen from the plane defined by its three substituent atoms. ${ }^{\mathrm{e}}$ this descriptor $(\tilde{\omega})$ is related to the capacity of an electrophile to promote a soft (covalent) reaction $\left[\tilde{\omega}=(I+A)^{2} / 8(I-A)=\left(E_{\mathrm{HOMO}}+E_{\mathrm{LUMO}}\right)^{2} / 8\left(E_{\mathrm{HOMO}}-E_{\mathrm{LUMO}}\right)[29]\right.$.

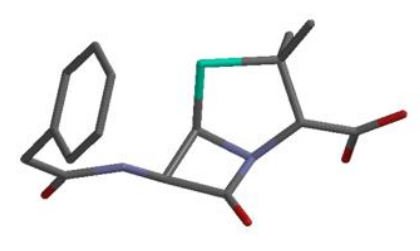

(A)

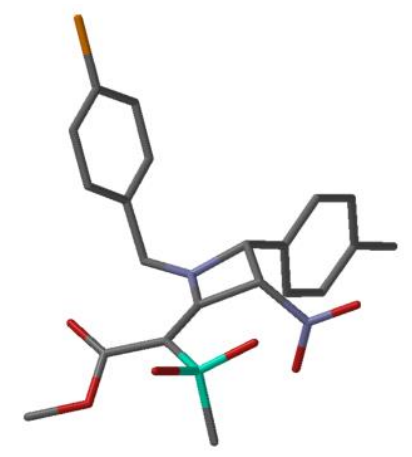

(B)

Figure 4. Cont. 


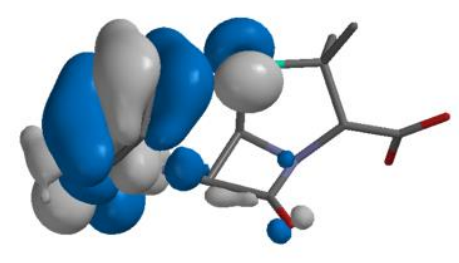

(C)

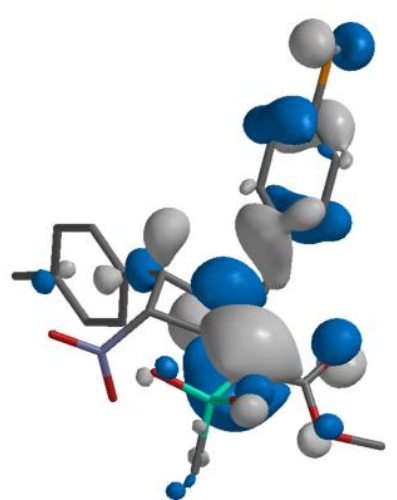

(D)

Figure 4. Geometry optimized models (DFT $\tilde{\omega}$ B97X-D/6-31G*) of the structures of penicillin G (A) and nitroazetidine 22 (B) and corresponding HOMO density maps ((C) and (D), respectively).

\section{Materials and Methods}

\subsection{Chemistry}

Materials and methods: ${ }^{1} \mathrm{H}$ NMR and ${ }^{13} \mathrm{C}$ NMR spectra were recorded with a Varian Mercury 300 Plus spectrometer, at 300 and $75 \mathrm{MHz}$, respectively, or with a Jeol JNMECZ400R, at 400 and $101 \mathrm{MHz}$, respectively; chemical shifts (TMS as internal reference) are reported as $\delta$ values (ppm). Signals are designated as follows: s, singlet; d, doublet; $\mathrm{dd}$, doublet of doublets; $\mathrm{t}$, triplet; q, quartet; $\mathrm{m}$, multiplet. Gas chromatography-mass spectrometry (GC-MS) was performed on a HP 5890/5971 (EI $70 \mathrm{eV})$ system equipped with a HP-1 MS capillary column $(12 \mathrm{~m} \times 0.2 \mathrm{~mm}$ i.d $\times 0.33 \mu \mathrm{m})$. High-resolution mass spectra (HRMS) were obtained with an Agilent MSD TOF mass spectrometer, and recorded in positive ion mode with an electrospray (ESI) source. Melting points were determined with a Büchi 535 apparatus and are uncorrected. IR spectra were recorded on a Perkin Elmer Spectrum 65 FT-IR and wave numbers are reported in $\mathrm{cm}^{-1}$. Petroleum ether and light petroleum refer to the fractions with bp $40-60^{\circ} \mathrm{C}$ and $80-100{ }^{\circ} \mathrm{C}$, respectively. Silica gel 230-400 mesh was used for column chromatography, all solvents being distilled before use. TLC analysis were performed on commercially prepared 60 F254 silica gel plates and visualized by UV irradiation (eluent: petroleum ether-ethyl acetate). Tetrahydrofuran (THF) dry was commercial. All other commercially available reagents were used as received.

The starting nitrothiophene 27, the 5-pyrrolidin-1-ylpentadienoate 28 , all the 2methylthio- and 2-methylsolfonyl-5-arylpentadienoates 29 and 30 have been already described [12,30]. Two new substrates, the 2-methylthio and 2-methylsolfonyl-5cyclohexylpentadienoates (29e and 30e, respectively), were prepared following the same procedure, and their characterization is reported below. Synthesis and characterization of azetidines 5-9, 12-14 have been already reported; [12] for the new azetidines 10, 11, 15-26, obtained following the same procedure, the characterization is reported below.

Typical procedure for the cyclization of methyl 2-methylsolfonyl-4-nitro-3phenylsolfonylpentadienoates to azetidines:

In a flask, the relevant nitropentadienoate $(0.15 \mathrm{mmol})$ was dissolved in DCM $(2.8 \mathrm{~mL})$ and the necessary amine ( 3.3 mol equiv.) was added by syringe under magnetic stirring. The reaction was followed by TLC and at the end, the solvent evaporated under reduced pressure. The crude was purified by chromatography on a silica gel column using petroleum ether/ethyl acetate mixtures as eluent to obtain the title compounds, as pure cis/trans diastereomeric mixtures (each diastereoisomer as racemic mixture), interconverting on time, to the final equilibrium ratios.

Characterization of new compounds (29e, 30e, 10, 11, 15-26):

Methyl (2E,4E)-5-cyclohexyl-2-(methylthio)-4-nitro-3-(phenylsulfonyl)penta-2,4-dienoate (29e). White solid. M.p. $192.5-193.2{ }^{\circ} \mathrm{C}$ (Ethanol). ${ }^{1} \mathrm{H}$ NMR $\left(\mathrm{CDCl}_{3}, 300 \mathrm{MHz}\right) \delta 0.99-1.41(4 \mathrm{H}$, $\mathrm{m}), 1.57-1.83(6 \mathrm{H}, \mathrm{m}), 2.10-2.29(1 \mathrm{H}, \mathrm{m}), 2.34(3 \mathrm{H}, \mathrm{s}), 4.06(3 \mathrm{H}, \mathrm{s}), 7.36(1 \mathrm{H}, \mathrm{d}, J 11.6 \mathrm{~Hz})$, 
7.42-7.56 (2H, m), 7.55-7.65 (1H, m), 7.83-7.95 (2H, m). ${ }^{13} \mathrm{C} \mathrm{NMR}\left(\mathrm{CDCl}_{3}, 101 \mathrm{MHz}\right) \delta$ $14.88,24.77,24.95,25.62,30.85,31.71,39.04,53.57,123.15,128.70,129.33,134.18,138.79$, 139.44, 151.97, 155.27, 163.21. HRMS (ESI) $m / z$ calcd $[\mathrm{M}+\mathrm{H}]^{+} \mathrm{C}_{19} \mathrm{H}_{24} \mathrm{NO}_{6} \mathrm{~S}_{2}{ }^{+} 426.1040$, found 426.1042 .

Methyl (2E,4E)-5-cyclohexyl-2-(methylsulfonyl)-4-nitro-3-(phenylsulfonyl)penta-2,4-dienoate (30e). White solid. ${ }^{1} \mathrm{H}$ NMR $\left(\mathrm{CDCl}_{3}, 300 \mathrm{MHz}\right) \delta 1.01-1.37(4 \mathrm{H}, \mathrm{m}), 1.74(6 \mathrm{H}, \mathrm{d}, J 14.2 \mathrm{~Hz}), 2.22-$ $2.40(1 \mathrm{H}, \mathrm{m}), 3.18(3 \mathrm{H}, \mathrm{s}), 4.09(3 \mathrm{H}, \mathrm{s}), 7.33(1 \mathrm{H}, \mathrm{d}, J 11.9 \mathrm{~Hz}), 7.56(2 \mathrm{H}, \mathrm{t}, J 7.9 \mathrm{~Hz}), 7.66-7.78$ (1H, m), 7.81-7.93 (2H, m). ${ }^{13} \mathrm{C} \mathrm{NMR}\left(\mathrm{CDCl}_{3}, 101 \mathrm{MHz}\right) \delta 24.67,24.88,25.59,30.56,30.68$, 39.15, 42.19, 54.77, 129.78, 130.02, 135.80, 135.86, 136.31, 141.03, 145.68, 151.83, 162.10. HRMS (ESI) $m / z[\mathrm{M}+\mathrm{H}]^{+} 458.0935$ (calcd for $\mathrm{C}_{19} \mathrm{H}_{24} \mathrm{NO}_{8} \mathrm{~S}_{2}{ }^{+}, 458.0938$ ).

Methyl (E)-2-(1-butyl-4-(4-(methylsulfonyl)phenyl)-3-nitroazetidin-2-ylidene)-2-(methylsulfonyl) acetate (10). Yellow solid. ${ }^{1} \mathrm{H}$ NMR $\left(\mathrm{CDCl}_{3}, 300 \mathrm{MHz}\right) \delta 0.80-0.98\left(3 \mathrm{H}_{\mathrm{A}}+3 \mathrm{H}_{\mathrm{B}}, \mathrm{m}\right), 1.25-1.42$ $\left(4 \mathrm{H}_{\mathrm{A}+\mathrm{B}}, \mathrm{m}\right), 1.42-1.62\left(4 \mathrm{H}_{\mathrm{A}+\mathrm{B}}, \mathrm{m}\right), 2.96-3.05\left(1 \mathrm{H}_{\mathrm{A}}\right.$ or $\left.1 \mathrm{H}_{\mathrm{B}}, \mathrm{m}\right), 3.08\left(3 \mathrm{H}_{\mathrm{A}}\right.$ or $\left.3 \mathrm{H}_{\mathrm{B}}, \mathrm{s}\right), 3.10$ $\left(3 \mathrm{H}_{\mathrm{A}}\right.$ or $\left.3 \mathrm{H}_{\mathrm{B}}, \mathrm{s}\right), 3.11\left(3 \mathrm{H}_{\mathrm{A}}\right.$ or $\left.3 \mathrm{H}_{\mathrm{B}}, \mathrm{s}\right), 3.14\left(3 \mathrm{H}_{\mathrm{A}}\right.$ or $\left.3 \mathrm{H}_{\mathrm{B}}, \mathrm{s}\right), 3.15-3.25\left(1 \mathrm{H}_{\mathrm{A}}\right.$ or $\left.1 \mathrm{H}_{\mathrm{B}}, \mathrm{m}\right), 3.87$ $\left(3 \mathrm{H}_{\mathrm{A}}\right.$ or $\left.3 \mathrm{H}_{\mathrm{B}}, \mathrm{s}\right), 3.89\left(3 \mathrm{H}_{\mathrm{A}}\right.$ or $\left.3 \mathrm{H}_{\mathrm{B}}, \mathrm{s}\right), 4.02-4.16\left(2 \mathrm{H}_{\mathrm{A}+\mathrm{B}}, \mathrm{m}\right), 5.24\left(1 \mathrm{H}_{\mathrm{A}}, \mathrm{d}, J 1.8 \mathrm{~Hz}\right), 5.62$ $\left(1 \mathrm{H}_{\mathrm{B}}, \mathrm{d}, J 6.0 \mathrm{~Hz}\right), 5.86\left(1 \mathrm{H}_{\mathrm{A}}, \mathrm{d}, J 1.9 \mathrm{~Hz}\right), 6.51\left(1 \mathrm{H}_{\mathrm{B}}, \mathrm{d}, J 6.0 \mathrm{~Hz}\right), 7.55\left(2 \mathrm{H}_{\mathrm{A}}\right.$ or $2 \mathrm{H}_{\mathrm{B}}, \mathrm{d}, J$ $8.3 \mathrm{~Hz}), 7.57\left(2 \mathrm{H}_{\mathrm{A}}\right.$ or $\left.2 \mathrm{H}_{\mathrm{B}}, \mathrm{d}, J 8.2 \mathrm{~Hz}\right), 7.98\left(2 \mathrm{H}_{\mathrm{A}}\right.$ or $\left.2 \mathrm{H}_{\mathrm{B}}, \mathrm{d}, J 8.3 \mathrm{~Hz}\right), 8.07\left(2 \mathrm{H}_{\mathrm{A}}\right.$ or $2 \mathrm{H}_{\mathrm{B}}, \mathrm{d}, J$ 8.2 Hz). ${ }^{13} \mathrm{C} \mathrm{NMR}\left(\mathrm{CDCl}_{3}, 101 \mathrm{MHz}\right) \delta 13.71,13.76,19.91,20.07,29.19,29.60,43.65,44.18$, $44.42,44.48,48.01,49.21,52.33,52.46,68.50,69.95,83.14,84.60,99.66,99.93,128.13,128.25$, $128.57,128.97,136.58,138.89,142.50,142.79,158.61,160.20,162.80,164.22$. HRMS (ESI) $\mathrm{m} / z$ $[\mathrm{M}+\mathrm{H}]^{+} 461.1044$ (calcd for $\mathrm{C}_{18} \mathrm{H}_{25} \mathrm{~N}_{2} \mathrm{O}_{8} \mathrm{~S}_{2}{ }^{+}, 461.1047$ ).

Methyl (E)-2-(1-benzyl-4-cyclohexyl-3-nitroazetidin-2-ylidene)-2-(methylsulfonyl)acetate (11). Orange solid. ${ }^{1} \mathrm{H}$ NMR $\left(\mathrm{CDCl}_{3}, 300 \mathrm{MHz}\right) \delta 0.82-1.36(4 \mathrm{H}, \mathrm{m}), 1.47-1.96(7 \mathrm{H}, \mathrm{m}), 3.09(3 \mathrm{H}, \mathrm{s})$, $3.70(3 \mathrm{H}, \mathrm{s}), 4.04(1 \mathrm{H}, \mathrm{dd}, J$ 4.9, $2.0 \mathrm{~Hz}), 4.36(1 \mathrm{H}, \mathrm{d}, J 15.8 \mathrm{~Hz}), 5.34(1 \mathrm{H}, \mathrm{d}, J 15.8 \mathrm{~Hz}), 5.92$ $(1 \mathrm{H}, \mathrm{d}, J 1.9 \mathrm{~Hz}), 7.29(2 \mathrm{H}, \mathrm{d}, J 1.9 \mathrm{~Hz}), 7.27-7.46(3 \mathrm{H}, \mathrm{m}) .{ }^{13} \mathrm{C} \mathrm{NMR}\left(\mathrm{CDCl}_{3}, 101 \mathrm{MHz}\right) \delta$ 25.34, 25.62, 25.93, 26.38, 28.26, 38.20, 44.17, 52.22, 72.96, 80.82, 98.83, 127.76, 128.43, 129.17, $133.73,158.80,163.24$ (two carbons accidentally isochronous). HRMS (ESI) $\mathrm{m} / z$ [M $+\mathrm{H}]^{+}$ 423.1581 (calcd for $\mathrm{C}_{20} \mathrm{H}_{27} \mathrm{~N}_{2} \mathrm{O}_{6} \mathrm{~S}^{+}$, 423.1584).

Methyl (E)-2-(1-benzyl-3-nitro-4-(thiophen-2-yl)azetidin-2-ylidene)-2-(methylsulfonyl)acetate (15). Whitish solid (taken up with petroleum ether). The sample analyzed at the NMR was a diastereomeric mixture (A, 43\%, cis; B 57\% trans). On time, A becomes the prevalent isomer. ${ }^{1} \mathrm{H} \mathrm{NMR}\left(\mathrm{CDCl}_{3}, 300 \mathrm{MHz}\right) \delta 3.15\left(3 \mathrm{H}_{\mathrm{B}}, \mathrm{s}\right), 3.16\left(3 \mathrm{H}_{\mathrm{A}}, \mathrm{s}\right), 3.76\left(3 \mathrm{H}_{\mathrm{B}}, \mathrm{s}\right), 3.83\left(3 \mathrm{H}_{\mathrm{A}}, \mathrm{s}\right), 4.19$ $\left(1 \mathrm{H}_{\mathrm{B}}, \mathrm{d}, J 15.6 \mathrm{~Hz}\right), 4.33\left(1 \mathrm{H}_{\mathrm{A}}, \mathrm{d}, J 15.0 \mathrm{~Hz}\right), 5.28\left(1 \mathrm{H}_{\mathrm{B}}, \mathrm{d}, J 2.0 \mathrm{~Hz}\right), 5.46-5.57\left(2 \mathrm{H}_{\mathrm{A}}+1 \mathrm{H}_{\mathrm{B}}\right.$, $\mathrm{m}), 6.08\left(1 \mathrm{H}_{\mathrm{B}}, \mathrm{d}, J 2.0 \mathrm{~Hz}\right), 6.40\left(1 \mathrm{H}_{\mathrm{A}}, \mathrm{d}, J 5.8 \mathrm{~Hz}\right), 7.01-7.10,7.11-7.22,7.28-7.38$ (in all, $6 \mathrm{H}_{\mathrm{A}}$ $\left.+6 \mathrm{H}_{\mathrm{B}}, \mathrm{m}\right), 7.42\left(1 \mathrm{H}_{\mathrm{A}}, \mathrm{d}, J 5.0 \mathrm{~Hz}\right), 7.49\left(1 \mathrm{H}_{\mathrm{B}}, \mathrm{d}, J 4.6 \mathrm{~Hz}\right) .{ }^{13} \mathrm{C} \mathrm{NMR}\left(\mathrm{CDCl}_{3}, 75 \mathrm{MHz}\right) \delta$ $43.58,44.04,51.24,51.76,52.21,52.23,63.48,65.70,84.52,86.02,99.63,100.31,127.82,127.92$, $127.94,128.33,128.56,128.63,128.75,129.02,129.04,129.06,129.11,129.40,131.78,133.23$, $133.47,135.04,158.56,158.75,162.95,163.13$. HRMS (ESI) $m / z[\mathrm{M}+\mathrm{H}]^{+} 423.0683$ (calcd for $\mathrm{C}_{18} \mathrm{H}_{19} \mathrm{~N}_{2} \mathrm{O}_{6} \mathrm{~S}_{2}{ }^{+}$, 423.0679).

Methyl (E)-2-(1-(2-chlorobenzyl)-3-nitro-4-(thiophen-2-yl)azetidin-2-ylidene)-2-(methylsulfonyl) acetate (16). Whitish solid. M.p. 65.1-83.4 (taken up with petroleum ether). The sample analyzed at the NMR was a diastereomeric mixture (A, 43\%, cis; B, 57\% trans). On time, A becomes the prevalent isomer. ${ }^{1} \mathrm{H}$ NMR $\left(\mathrm{CDCl}_{3}, 300 \mathrm{MHz}\right) \delta 3.16\left(3 \mathrm{H}_{\mathrm{A}}+3 \mathrm{H}_{\mathrm{B}}, \mathrm{s}\right), 3.70$ $\left(3 \mathrm{H}_{\mathrm{B}}, \mathrm{s}\right), 3.78\left(3 \mathrm{H}_{\mathrm{A}}, \mathrm{s}\right), 4.42\left(1 \mathrm{H}_{\mathrm{B}}, \mathrm{d}, J 16.2 \mathrm{~Hz}\right), 4.68\left(1 \mathrm{H}_{\mathrm{A}}, \mathrm{d}, J 15.5 \mathrm{~Hz}\right), 5.28\left(1 \mathrm{H}_{\mathrm{B}}, \mathrm{d}, J\right.$ $1.9 \mathrm{~Hz}), 5.32-5.44\left(1 \mathrm{H}_{\mathrm{A}}+1 \mathrm{H}_{\mathrm{B}}, \mathrm{m}\right), 5.50\left(1 \mathrm{H}_{\mathrm{A}}, \mathrm{d}, J 5.9 \mathrm{~Hz}\right), 6.07\left(1 \mathrm{H}_{\mathrm{B}}, \mathrm{d}, J 1.9 \mathrm{~Hz}\right), 6.46$ $\left(1 \mathrm{H}_{\mathrm{A}}, \mathrm{d}, J 5.8 \mathrm{~Hz}\right), 6.98-7.38\left(7 \mathrm{H}_{\mathrm{A}}+6 \mathrm{H}_{\mathrm{B}}, \mathrm{m}\right), 7.46\left(1 \mathrm{H}_{\mathrm{B}}, \mathrm{dd}, J 4.5,1.8 \mathrm{~Hz}\right) .{ }^{13} \mathrm{C} \mathrm{NMR}$ $\left(\mathrm{CDCl}_{3}, 75 \mathrm{MHz}\right) \delta 43.53,44.06,49.19,50.22,52.16,64.46,66.28,84.15,86.15,100.26,100.71$, $127.36,127.40,127.70,127.93,128.41,128.73,128.87,129.33,129.44,129.75,129.92,129.96$, $130.10,130.61,130.80,131.16,131.98,133.34,133.83,135.08,158.46,159.56,162.92,162.95$ (two carbons accidentally isochronous). HRMS (ESI) $m / z[\mathrm{M}+\mathrm{H}]^{+} 457.0285$ (calcd for $\left.\mathrm{C}_{18} \mathrm{H}_{18} \mathrm{ClN}_{2} \mathrm{O}_{6} \mathrm{~S}_{2}{ }^{+}, 457.0289\right)$. 
Methyl (E)-2-(1-(3-chlorobenzyl)-3-nitro-4-(thiophen-2-yl)azetidin-2-ylidene)-2-(methylsulfonyl) acetate (17). Whitish solid. M.p. 63.2-84.0 (taken up with petroleum ether). The sample analyzed at the NMR was a diastereomeric mixture (A, 43\%, cis; B, 57\% trans). On time, A becomes the prevalent isomer. ${ }^{1} \mathrm{H} \mathrm{NMR}\left(\mathrm{CDCl}_{3}, 300 \mathrm{MHz}\right) \delta 3.13\left(3 \mathrm{H}_{\mathrm{B}}, \mathrm{s}\right), 3.15\left(3 \mathrm{H}_{\mathrm{A}}\right.$, $\mathrm{s}), 3.76\left(3 \mathrm{H}_{\mathrm{B}}, \mathrm{s}\right), 3.81\left(3 \mathrm{H}_{\mathrm{A}}, \mathrm{s}\right), 4.19\left(1 \mathrm{H}_{\mathrm{B}}, \mathrm{d}, J 15.7 \mathrm{~Hz}\right), 4.35\left(1 \mathrm{H}_{\mathrm{A}}, \mathrm{d}, J 15.2 \mathrm{~Hz}\right), 5.32\left(1 \mathrm{H}_{\mathrm{B}}\right.$, $\mathrm{d}, J 2.1 \mathrm{~Hz}), 5.39-5.51\left(1 \mathrm{H}_{\mathrm{A}}+1 \mathrm{H}_{\mathrm{B}}, \mathrm{m}\right), 5.54\left(1 \mathrm{H}_{\mathrm{A}}, \mathrm{d}, J 5.8 \mathrm{~Hz}\right), 6.09\left(1 \mathrm{H}_{\mathrm{B}}, \mathrm{d}, J 2.0 \mathrm{~Hz}\right), 6.43$ $\left(1 \mathrm{H}_{\mathrm{A}}, \mathrm{d}, J 5.8 \mathrm{~Hz}\right), 7.00-7.30\left(6 \mathrm{H}_{\mathrm{A}}+6 \mathrm{H}_{\mathrm{B}}, \mathrm{m}\right), 7.42\left(1 \mathrm{H}_{\mathrm{A}}, \mathrm{dd}, J 5.0,1.3 \mathrm{~Hz}\right), 7.48-7.50\left(1 \mathrm{H}_{\mathrm{B}}\right.$, m). ${ }^{13} \mathrm{C} \mathrm{NMR}\left(\mathrm{CDCl}_{3}, 101 \mathrm{MHz}\right) \delta 43.77,44.15,50.74,51.14,52.46,63.94,66.11,84.94,86.10$, $100.13,100.77,126.03,126.71,128.02,128.15,128.17,128.64,128.75,128.89,128.92,129.11$, 129.37, 129.78, 130.48, 131.62, 134.84, 134.99, 135.37, 135.75, 158.64, 158.73, 163.01, 163.26 (three carbons accidentally isochronous). HRMS (ESI) $m / z[\mathrm{M}+\mathrm{H}]^{+} 457.0288$ (calcd for $^{\circ}$ $\mathrm{C}_{18} \mathrm{H}_{18} \mathrm{ClN}_{2} \mathrm{O}_{6} \mathrm{~S}_{2}{ }^{+}$, 457.0289).

Methyl (E)-2-(1-(4-chlorobenzyl)-3-nitro-4-(thiophen-2-yl)azetidin-2-ylidene)-2-(methylsulfonyl) acetate (18). Whitish solid (taken up with petroleum ether). The sample analyzed at the NMR was a diastereomeric mixture (A, 42\%, cis; B, 58\% trans). On time, A becomes the prevalent isomer. ${ }^{1} \mathrm{H} \mathrm{NMR}\left(\mathrm{CDCl}_{3}, 300 \mathrm{MHz}\right) \delta 3.14\left(3 \mathrm{H}_{\mathrm{B}}, \mathrm{s}\right), 3.16\left(3 \mathrm{H}_{\mathrm{A}}, \mathrm{s}\right), 3.78\left(3 \mathrm{H}_{\mathrm{B}}\right.$, s), $3.84\left(3 \mathrm{H}_{\mathrm{A}}, \mathrm{s}\right), 4.18\left(1 \mathrm{H}_{\mathrm{B}}, \mathrm{d}, J 15.6 \mathrm{~Hz}\right), 4.32\left(1 \mathrm{H}_{\mathrm{A}}, \mathrm{d}, J 15.0 \mathrm{~Hz}\right), 5.27\left(1 \mathrm{H}_{\mathrm{B}}, \mathrm{d}, J 2.0 \mathrm{~Hz}\right)$, 5.38-5.64 $\left(2 \mathrm{H}_{\mathrm{A}}+1 \mathrm{H}_{\mathrm{B}}, \mathrm{m}\right), 6.09\left(1 \mathrm{H}_{\mathrm{B}}, \mathrm{d}, J 2.0 \mathrm{~Hz}\right), 6.41\left(1 \mathrm{H}_{\mathrm{A}}, \mathrm{d}, J 5.7 \mathrm{~Hz}\right), 6.99-7.42\left(6 \mathrm{H}_{\mathrm{A}}\right.$ $\left.+6 \mathrm{H}_{\mathrm{B}}, \mathrm{m}\right), 7.43\left(1 \mathrm{H}_{\mathrm{A}}, \mathrm{d}, J 5.1 \mathrm{~Hz}\right), 7.49\left(1 \mathrm{H}_{\mathrm{B}}, \mathrm{d}, J 4.4 \mathrm{~Hz}\right) .{ }^{13} \mathrm{C} \mathrm{NMR}\left(\mathrm{CDCl}_{3}, 75 \mathrm{MHz}\right) \delta$ $43.71,44.13,50.69,51.05,52.43,52.46,63.59,65.85,84.79,86.07,99.93,100.68,128.02,128.13$, $128.86,129.06,129.28,129.37,129.41,129.43,129.68,130.08,131.63,131.85,132.19,134.44$, $134.78,134.87,158.65,158.80,163.07,163.32$. HRMS (ESI) $m / z[M+H]^{+} 457.0293$ (calcd for $\mathrm{C}_{18} \mathrm{H}_{18} \mathrm{ClN}_{2} \mathrm{O}_{6} \mathrm{~S}_{2}{ }^{+}, 457.0289$.

Methyl (E)-2-(1-(3,4-dichlorobenzyl)-3-nitro-4-(thiophen-2-yl)azetidin-2-ylidene)-2-(methylsulfonyl) acetate (19). Whitish solid. M.p. 69.8-73.7 (taken up with petroleum ether). The sample analyzed at the NMR was a diastereomeric mixture (A, 47\%, cis; B, 53\% trans). On time, A becomes the prevalent isomer. ${ }^{1} \mathrm{H}$ NMR $\left(\mathrm{CDCl}_{3}, 300 \mathrm{MHz}\right) \delta 3.13\left(3 \mathrm{H}_{\mathrm{B}}, \mathrm{s}\right), 3.15\left(3 \mathrm{H}_{\mathrm{A}}, \mathrm{s}\right)$, $3.78\left(3 \mathrm{H}_{\mathrm{B}}, \mathrm{s}\right), 3.83\left(3 \mathrm{H}_{\mathrm{A}}, \mathrm{s}\right), 4.19\left(1 \mathrm{H}_{\mathrm{B}}, \mathrm{d}, J 15.7 \mathrm{~Hz}\right), 4.35\left(1 \mathrm{H}_{\mathrm{A}}, \mathrm{d}, J 15.1 \mathrm{~Hz}\right), 5.32\left(1 \mathrm{H}_{\mathrm{B}}, \mathrm{d}, J\right.$ $2.0 \mathrm{~Hz}), 5.44\left(1 \mathrm{H}_{\mathrm{A}}+1 \mathrm{H}_{\mathrm{B}}, \mathrm{m}\right), 5.53\left(1 \mathrm{H}_{\mathrm{A}}, \mathrm{d}, J 5.5 \mathrm{~Hz}\right), 6.10\left(1 \mathrm{H}_{\mathrm{B}}, \mathrm{d}, J 2.0 \mathrm{~Hz}\right), 6.44\left(1 \mathrm{H}_{\mathrm{A}}, \mathrm{d}, J\right.$ $5.6 \mathrm{~Hz}), 6.97-7.53\left(6 \mathrm{H}_{\mathrm{A}}+6 \mathrm{H}_{\mathrm{B}}, \mathrm{m}\right) .{ }^{13} \mathrm{C} \mathrm{NMR}\left(\mathrm{CDCl}_{3}, 75 \mathrm{MHz}\right) \delta 43.69,44.05,50.13,50.46$, $52.40,63.87,66.06,84.93,85.98,100.30,100.96,127.15,127.82,127.95,128.10,128.91,129.10$, $129.33,129.71,129.93,130.55,131.02,131.05,131.38,132.60,132.91,133.16,133.18,133.48$, $133.93,134.61,158.43,158.71,162.93,163.21$ (two carbons accidentally isochronous). HRMS (ESI) $m / z[\mathrm{M}+\mathrm{H}]^{+} 490.9898$ (calcd for $\mathrm{C}_{18} \mathrm{H}_{17} \mathrm{Cl}_{2} \mathrm{~N}_{2} \mathrm{O}_{6} \mathrm{~S}_{2}{ }^{+}, 490.9900$ ).

Methyl (E)-2-(1-(2-chlorobenzyl)-3-nitro-4-(p-tolyl)azetidin-2-ylidene)-2-(methylsulfonyl)acetate (20). Whitish solid. M.p. 66.2-74.2 (taken up with petroleum ether). The sample analyzed at the NMR was a diastereomeric mixture (A, 45\%, cis; B 55\% trans). On time, A becomes the prevalent isomer. ${ }^{1} \mathrm{H}$ NMR $\left(\mathrm{CDCl}_{3}, 300 \mathrm{MHz}\right) \delta 2.32\left(3 \mathrm{H}_{\mathrm{A}}, \mathrm{s}\right), 2.38\left(3 \mathrm{H}_{\mathrm{B}}, \mathrm{s}\right), 3.13$ $\left(3 \mathrm{H}_{\mathrm{A}}, \mathrm{s}\right), 3.15\left(3 \mathrm{H}_{\mathrm{B}}, \mathrm{s}\right), 3.70\left(3 \mathrm{H}_{\mathrm{B}}, \mathrm{s}\right), 3.82\left(3 \mathrm{H}_{\mathrm{A}}, \mathrm{s}\right), 4.28\left(1 \mathrm{H}_{\mathrm{B}}, \mathrm{d}, J 16.1 \mathrm{~Hz}\right), 4.57\left(1 \mathrm{H}_{\mathrm{A}}, \mathrm{d}\right.$, $J 15.4 \mathrm{~Hz}), 4.93\left(1 \mathrm{H}_{\mathrm{B}}, \mathrm{d}, J 1.9 \mathrm{~Hz}\right), 5.21\left(1 \mathrm{H}_{\mathrm{A}}, \mathrm{d}, J 6.0 \mathrm{~Hz}\right), 5.34-5.53\left(1 \mathrm{H}_{\mathrm{A}}+1 \mathrm{H}_{\mathrm{B}}, \mathrm{m}\right), 5.94$ $\left(1 \mathrm{H}_{\mathrm{B}}, \mathrm{d}, J 1.9 \mathrm{~Hz}\right), 6.41\left(1 \mathrm{H}_{\mathrm{A}}, \mathrm{d}, J 5.9 \mathrm{~Hz}\right), 7.05-7.35\left(8 \mathrm{H}_{\mathrm{A}}+8 \mathrm{H}_{\mathrm{B}}, \mathrm{m}\right) .{ }^{13} \mathrm{C} \mathrm{NMR}\left(\mathrm{CDCl}_{3}\right.$, $75 \mathrm{MHz}) \delta 21.30,21.33,43.45,44.06,49.26,50.43,52.11,68.82,70.95,83.40,85.44,99.69$, $100.07,126.76,127.02,127.12,127.30,127.38,129.17,129.46,129.68,129.71,129.92,130.15$, 130.26, 130.45, 130.77, 130.87, 131.22, 133.35, 133.96, 140.58, 140.99, 159.00, 160.71, 163.09, 163.1421 (two carbons accidentally isochronous). HRMS (ESI) $m / z[\mathrm{M}+\mathrm{H}]^{+} 465.0885$ (calcd for $\mathrm{C}_{21} \mathrm{H}_{22} \mathrm{ClN}_{2} \mathrm{O}_{6} \mathrm{~S}^{+}$, 465.0882).

Methyl (E)-2-(1-(3-chlorobenzyl)-3-nitro-4-(p-tolyl)azetidin-2-ylidene)-2-(methylsulfonyl)acetate (21). Whitish solid. M.p. 67.3-78.7 (taken up with petroleum ether). The sample analyzed at the NMR was a diastereomeric mixture (A, 37\%, cis; B 63\% trans). On time, A becomes the prevalent isomer. ${ }^{1} \mathrm{H} \mathrm{NMR}\left(\mathrm{CDCl}_{3}, 300 \mathrm{MHz}\right) \delta 2.35\left(3 \mathrm{H}_{\mathrm{A}} \mathrm{s}\right), 2.39\left(3 \mathrm{H}_{\mathrm{B}}, \mathrm{s}\right), 3.13\left(3 \mathrm{H}_{\mathrm{A}}+\right.$ $\left.3 \mathrm{H}_{\mathrm{B}}, \mathrm{s}\right), 3.76\left(3 \mathrm{H}_{\mathrm{B}}, \mathrm{s}\right), 3.85\left(3 \mathrm{H}_{\mathrm{A}}, \mathrm{s}\right), 4.07\left(1 \mathrm{H}_{\mathrm{B}}, \mathrm{d}, J 15.6 \mathrm{~Hz}\right), 4.25\left(1 \mathrm{H}_{\mathrm{A}}, \mathrm{d}, J 15.0 \mathrm{~Hz}\right), 4.99$ $\left(1 \mathrm{H}_{\mathrm{B}}, \mathrm{d}, J 2.0 \mathrm{~Hz}\right), 5.25\left(1 \mathrm{H}_{\mathrm{A}}, \mathrm{d}, J 5.9 \mathrm{~Hz}\right), 5.46\left(1 \mathrm{H}_{\mathrm{B}}, \mathrm{d}, J 15.6 \mathrm{~Hz}\right), 5.55\left(1 \mathrm{H}_{\mathrm{A}}, \mathrm{d}, J 15.0 \mathrm{~Hz}\right)$, $5.98\left(1 \mathrm{H}_{\mathrm{B}}, \mathrm{d}, J 1.9 \mathrm{~Hz}\right), 6.40\left(1 \mathrm{H}_{\mathrm{A}}, \mathrm{d}, J 5.9 \mathrm{~Hz}\right), 7.02-7.29\left(8 \mathrm{H}_{\mathrm{A}}+8 \mathrm{H}_{\mathrm{B}}, \mathrm{m}\right) .{ }^{13} \mathrm{C} \mathrm{NMR}\left(\mathrm{CDCl}_{3}\right.$, 
$75 \mathrm{MHz}) \delta 21.31,21.34,43.57,44.03,50.69,51.29,52.26,68.21,70.75,83.99,85.25,99.55$, $100.21,125.95,126.31,126.69,127.20,127.37,128.10,128.44,128.74,128.78,128.84,129.92$, $130.31,130.37,130.41,134.82,134.88,135.30,135.67,140.90,141.22,159.16,159.66,163.05$, 163.31 (two carbons are accidentally isochronous). HRMS (ESI) $m / z[\mathrm{M}+\mathrm{H}]^{+} 465.0883$ (calcd for $\mathrm{C}_{21} \mathrm{H}_{22} \mathrm{ClN}_{2} \mathrm{O}_{6} \mathrm{~S}^{+}$, 465.0882).

Methyl (E)-2-(1-(4-chlorobenzyl)-3-nitro-4-(p-tolyl)azetidin-2-ylidene)-2-(methylsulfonyl)acetate (22). Pale yellow solid. ${ }^{1} \mathrm{H}$ NMR $\left(\mathrm{CDCl}_{3}, 300 \mathrm{MHz}\right) \delta 2.35\left(3 \mathrm{H}_{\mathrm{A}}, \mathrm{s}\right), 2.40\left(3 \mathrm{H}_{\mathrm{B}}, \mathrm{s}\right), 3.14$ $\left(3 \mathrm{H}_{\mathrm{A}}+3 \mathrm{H}_{\mathrm{B}}, \mathrm{s}\right), 3.78\left(3 \mathrm{H}_{\mathrm{B}}, \mathrm{s}\right), 3.86\left(3 \mathrm{H}_{\mathrm{A}}, \mathrm{s}\right), 4.05\left(1 \mathrm{H}_{\mathrm{B}}, \mathrm{d}, J 15.5 \mathrm{~Hz}\right), 4.23\left(1 \mathrm{H}_{\mathrm{A}}, \mathrm{d}, J 15.0 \mathrm{~Hz}\right)$, $4.93\left(1 \mathrm{H}_{\mathrm{B}}, \mathrm{d}, J 1.9 \mathrm{~Hz}\right), 5.20\left(1 \mathrm{H}_{\mathrm{A}}, \mathrm{d}, J 6.0 \mathrm{~Hz}\right), 5.50\left(1 \mathrm{H}_{\mathrm{B}}, \mathrm{d}, J 15.5 \mathrm{~Hz}\right), 5.59\left(1 \mathrm{H}_{\mathrm{A}}, \mathrm{d}, J\right.$ $15.0 \mathrm{~Hz}), 5.97\left(1 \mathrm{H}_{\mathrm{B}}, \mathrm{d}, J 1.9 \mathrm{~Hz}\right), 6.38\left(1 \mathrm{H}_{\mathrm{A}}, \mathrm{d}, J 5.9 \mathrm{~Hz}\right), 6.85-7.45\left(8 \mathrm{H}_{\mathrm{A}}+8 \mathrm{H}_{\mathrm{B}}, \mathrm{m}\right) .{ }^{13} \mathrm{C} \mathrm{NMR}$ $\left(\mathrm{CDCl}_{3}, 75 \mathrm{MHz}\right) \delta 21.32,21.34,43.52,44.04,50.61,51.21,52.24,67.90,70.47,83.89,85.24$, $99.32,100.04,127.18,127.34,129.20,129.28,129.35,129.92,130.08,130.36,130.55,131.07$, $131.75,132.11,134.25,134.66,140.89,141.19,159.24,159.69,163.12,163.35$ (two carbons are accidentally isochronous). HRMS (ESI) $\mathrm{m} / z[\mathrm{M}+\mathrm{H}]^{+} 465.0880$ (calcd for $\mathrm{C}_{21} \mathrm{H}_{22} \mathrm{ClN}_{2} \mathrm{O}_{6} \mathrm{~S}^{+}$, 465.0882).

Methyl (E)-2-(1-(3,4-dichlorobenzyl)-3-nitro-4-(p-tolyl)azetidin-2-ylidene)-2-(methylsulfonyl)acetate (23). Whitish solid. M.p. 68.2-73.1 (taken up with petroleum ether). The sample analyzed at the NMR was a diastereomeric mixture (A, 37\%, cis; B 63\% trans). On time, A becomes the prevalent isomer. ${ }^{1} \mathrm{H}$ NMR $\left(\mathrm{CDCl}_{3}, 300 \mathrm{MHz}\right) \delta 2.35\left(3 \mathrm{H}_{\mathrm{A}}, \mathrm{s}\right), 2.40\left(3 \mathrm{H}_{\mathrm{B}}, \mathrm{s}\right), 3.13\left(3 \mathrm{H}_{\mathrm{B}}\right.$, $\mathrm{s}), 3.14\left(3 \mathrm{H}_{\mathrm{A}}, \mathrm{s}\right), 3.78\left(3 \mathrm{H}_{\mathrm{B}}, \mathrm{s}\right), 3.85\left(3 \mathrm{H}_{\mathrm{A}}, \mathrm{s}\right), 4.07\left(1 \mathrm{H}_{\mathrm{B}}, \mathrm{d}, J 15.6 \mathrm{~Hz}\right), 4.26\left(1 \mathrm{H}_{\mathrm{A}}, \mathrm{d}, J 15.1\right.$ $\mathrm{Hz}), 4.98\left(1 \mathrm{H}_{\mathrm{B}}, \mathrm{d}, J 1.9 \mathrm{~Hz}\right), 5.25\left(1 \mathrm{H}_{\mathrm{A}}, \mathrm{d}, J 5.9 \mathrm{~Hz}\right), 5.44\left(1 \mathrm{H}_{\mathrm{B}}, \mathrm{d}, J 15.6 \mathrm{~Hz}\right), 5.52\left(1 \mathrm{H}_{\mathrm{A}}\right.$, $\mathrm{d}, J 15.2 \mathrm{~Hz}), 5.99\left(1 \mathrm{H}_{\mathrm{B}}, \mathrm{d}, J 1.9 \mathrm{~Hz}\right), 6.41\left(1 \mathrm{H}_{\mathrm{A}}, \mathrm{d}, J 5.9 \mathrm{~Hz}\right), 6.92-7.06\left(2 \mathrm{H}_{\mathrm{A}}+2 \mathrm{H}_{\mathrm{B}}, \mathrm{m}\right)$, 7.07-7.31 $\left(10 \mathrm{H}_{\mathrm{A}}+10 \mathrm{H}_{\mathrm{B}}, \mathrm{m}\right), 7.32-7.47\left(2 \mathrm{H}_{\mathrm{A}}+2 \mathrm{H}_{\mathrm{B}}, \mathrm{m}\right) .{ }^{13} \mathrm{C} \mathrm{NMR}\left(\mathrm{CDCl}_{3}, 75 \mathrm{MHz}\right) \delta 21.31$, 21.34, 43.59, 44.04, 50.18, 50.72, 52.33, 68.27, 70.80, 84.09, 85.21, 99.74, 100.42, 126.19, 127.20, $127.22,127.38,127.95,128.67,129.96,130.00,130.41,130.46,130.67,130.99,132.50,132.89$, 133.07, 133.13, 133.50, 133.95, 141.02, 141.35, 159.28, 159.59, 163.09, 163.38 (two carbons are accidentally isochronous). HRMS (ESI) $\mathrm{m} / \mathrm{z}[\mathrm{M}+\mathrm{H}]^{+} 499.0495$ (calcd for $\mathrm{C}_{21} \mathrm{H}_{21} \mathrm{Cl}_{2} \mathrm{~N}_{2} \mathrm{O}_{6} \mathrm{~S}^{+}$, 499.0492).

Methyl (E)-2-(1-benzyl-4-(4-(methylsulfonyl)phenyl)-3-nitroazetidin-2-ylidene)-2-(methylsulfonyl) acetate (24). Viscous yellow oil. ${ }^{1} \mathrm{H} \mathrm{NMR}\left(\mathrm{CDCl}_{3}, 300 \mathrm{MHz}\right) \delta 3.07\left(3 \mathrm{H}_{\mathrm{B}}, \mathrm{s}\right), 3.08\left(3 \mathrm{H}_{\mathrm{A}}\right.$, $\mathrm{s}), 3.14\left(3 \mathrm{H}_{\mathrm{B}}, \mathrm{s}\right), 3.15\left(3 \mathrm{H}_{\mathrm{A}}, \mathrm{s}\right), 3.81\left(3 \mathrm{H}_{\mathrm{A}}, \mathrm{s}\right), 3.89\left(3 \mathrm{H}_{\mathrm{B}}, \mathrm{s}\right), 4.20\left(1 \mathrm{H}_{\mathrm{A}}, \mathrm{d}, J 15.4 \mathrm{~Hz}\right), 4.34$ $\left(1 \mathrm{H}_{\mathrm{B}}, \mathrm{d}, J 14.9 \mathrm{~Hz}\right), 5.06\left(1 \mathrm{H}_{\mathrm{A}}, \mathrm{d}, J 1.9 \mathrm{~Hz}\right), 5.32\left(1 \mathrm{H}_{\mathrm{B}}, \mathrm{d}, J 6.2 \mathrm{~Hz}\right), 5.45\left(1 \mathrm{H}_{\mathrm{A}}, \mathrm{d}, J 15.4 \mathrm{~Hz}\right)$, $5.52\left(1 \mathrm{H}_{\mathrm{B}}, \mathrm{d}, J 14.9 \mathrm{~Hz}\right), 5.93\left(1 \mathrm{H}_{\mathrm{A}}, \mathrm{d}, J 1.9 \mathrm{~Hz}\right), 6.45\left(1 \mathrm{H}_{\mathrm{B}}, \mathrm{d}, J 6.2 \mathrm{~Hz}\right), 7.03-7.16\left(4 \mathrm{H}_{\mathrm{A}}+\mathrm{B}\right.$, $\mathrm{m}), 7.27-7.38\left(6 \mathrm{H}_{\mathrm{A}+\mathrm{B}}, \mathrm{m}\right), 7.38-7.49\left(4 \mathrm{H}_{\mathrm{A}+\mathrm{B}}, \mathrm{m}\right), 7.94\left(2 \mathrm{H}_{\mathrm{B}}, \mathrm{d}, J 8.4 \mathrm{~Hz}\right), 8.01\left(2 \mathrm{H}_{\mathrm{A}}, \mathrm{d}, J\right.$ $8.3 \mathrm{~Hz}) .{ }^{13} \mathrm{C} \mathrm{NMR}\left(\mathrm{CDCl}_{3}, 101 \mathrm{MHz}\right) \delta 43.55,44.02,44.23,44.29,52.00,52.32,52.34,52.78$, $67.12,69.20,83.06,84.43,100.14,100.59,127.99,128.10,128.15,128.44,128.58,128.65,128.75$, 128.83, 129.09, 129.13, 132.51, 132.66, 136.08, 138.45, 142.23, 142.51, 158.58, 159.77, 162.82, 162.89. HRMS (ESI) $m / z[\mathrm{M}+\mathrm{H}]^{+} 495.0894$ (calcd for $\mathrm{C}_{21} \mathrm{H}_{23} \mathrm{~N}_{2} \mathrm{O}_{8} \mathrm{~S}_{2}{ }^{+}$, 495.0890).

Methyl (E)-2-(1-benzyl-4-(3,5-bis(trifluoromethyl)phenyl)-3-nitroazetidin-2-ylidene)-2-(methylsulfonyl)acetate (25). Yellow oil. ${ }^{1} \mathrm{H} \mathrm{NMR}\left(\mathrm{CDCl}_{3}, 300 \mathrm{MHz}\right) \delta 3.17\left(3 \mathrm{H}_{\mathrm{A}}\right.$ or $\left.3 \mathrm{H}_{\mathrm{B}}, \mathrm{s}\right), 3.18\left(3 \mathrm{H}_{\mathrm{A}}\right.$ or $\left.3 \mathrm{H}_{\mathrm{B}}, \mathrm{s}\right), 3.85\left(3 \mathrm{H}_{\mathrm{A}}\right.$ or $\left.3 \mathrm{H}_{\mathrm{B}}, \mathrm{s}\right), 3.92\left(3 \mathrm{H}_{\mathrm{A}}\right.$ or $\left.3 \mathrm{H}_{\mathrm{B}}, \mathrm{s}\right), 4.38\left(1 \mathrm{H}_{\mathrm{A}}\right.$ or $\left.1 \mathrm{H}_{\mathrm{B}}, \mathrm{d}, J 15.2 \mathrm{~Hz}\right), 4.49$ $\left(1 \mathrm{H}_{\mathrm{A}}\right.$ or $\left.1 \mathrm{H}_{\mathrm{B}}, \mathrm{d}, J 14.8 \mathrm{~Hz}\right), 5.10\left(1 \mathrm{H}_{\mathrm{A}}, \mathrm{d}, J 2.0 \mathrm{~Hz}\right), 5.28-5.40\left(2 \mathrm{H}, 1 \mathrm{H}_{\mathrm{B}}+1 \mathrm{H}_{\mathrm{A}}\right.$ or $\left.1 \mathrm{H}_{\mathrm{B}} \mathrm{m}\right)$, $5.44\left(1 \mathrm{H}_{\mathrm{A}}\right.$ or $\left.1 \mathrm{H}_{\mathrm{B}}, \mathrm{d}, J 14.9 \mathrm{~Hz}\right), 5.98\left(1 \mathrm{H}_{\mathrm{A}}, \mathrm{d}, J 2.0 \mathrm{~Hz}\right), 6.48\left(1 \mathrm{H}_{\mathrm{B}}, \mathrm{d}, J 6.1 \mathrm{~Hz}\right), 7.03-7.13$ $\left(4 \mathrm{H}_{\mathrm{A}}+4 \mathrm{H}_{\mathrm{B}}, \mathrm{m}\right), 7.26-7.36\left(6 \mathrm{H}_{\mathrm{A}}+6 \mathrm{H}_{\mathrm{B}}, \mathrm{m}\right), 7.58\left(2 \mathrm{H}_{\mathrm{A}}\right.$ or $\left.2 \mathrm{H}_{\mathrm{B}}, \mathrm{s}\right), 7.64\left(2 \mathrm{H}_{\mathrm{A}}\right.$ or $\left.2 \mathrm{H}_{\mathrm{B}}, \mathrm{s}\right), 7.89$ $\left(1 \mathrm{H}_{\mathrm{A}}\right.$ or $\left.1 \mathrm{H}_{\mathrm{B}}, \mathrm{s}\right), 7.94\left(1 \mathrm{H}_{\mathrm{A}}\right.$ or $\left.1 \mathrm{H}_{\mathrm{B}}, \mathrm{s}\right) .{ }^{13} \mathrm{C} \mathrm{NMR}\left(\mathrm{CDCl}_{3}, 101 \mathrm{MHz}\right) \delta 43.73,52.58,53.48$, 67.16, 83.19, 101.56, 122.75 (q, J 274.72 Hz), 124.38 (app t), 127.69, 129.08, 129.13, 129.29, $132.50,132.51$ (q, J 34.34 Hz), 133.32, 159.96, 162.94. HRMS (ESI) $m / z[M+\mathrm{H}]^{+} 553.0860$ (calcd for $\mathrm{C}_{22} \mathrm{H}_{19} \mathrm{~F}_{6} \mathrm{~N}_{2} \mathrm{O}_{6} \mathrm{~S}^{+}$, 553.0863).

Methyl (E)-2-(4-(3,5-bis(trifluoromethyl)phenyl)-1-(4-chlorobenzyl)-3-nitroazetidin-2-ylidene)-2(methylsulfonyl)acetate (26). Whitish solid (taken up with petroleum ether). The sample analyzed at the NMR was a diastereomeric mixture $(\mathrm{A}, 67 \%$, cis; $\mathrm{B}, 33 \%$ trans $) .{ }^{1} \mathrm{H}$ NMR $\left(\mathrm{CDCl}_{3}, 300 \mathrm{MHz}\right) \delta 3.16\left(3 \mathrm{H}_{\mathrm{A}}, \mathrm{s}\right), 3.17\left(3 \mathrm{H}_{\mathrm{B}}, \mathrm{s}\right), 3.84\left(3 \mathrm{H}_{\mathrm{B}}, \mathrm{s}\right), 3.92\left(3 \mathrm{H}_{\mathrm{A}}, \mathrm{s}\right), 4.32\left(1 \mathrm{H}_{\mathrm{B}}, \mathrm{d}, J\right.$ 
$15.4 \mathrm{~Hz}), 4.47\left(1 \mathrm{H}_{\mathrm{A}}, \mathrm{d}, J 14.9 \mathrm{~Hz}\right), 5.13\left(1 \mathrm{H}_{\mathrm{B}}, \mathrm{d}, J 2.0 \mathrm{~Hz}\right), 5.32-5.48\left(2 \mathrm{H}_{\mathrm{A}}+1 \mathrm{H}_{\mathrm{B}}, \mathrm{m}\right), 6.00$ $\left(1 \mathrm{H}_{\mathrm{B}}, \mathrm{d}, J 2.0 \mathrm{~Hz}\right), 6.50\left(1 \mathrm{H}_{\mathrm{A}}, \mathrm{d}, J 5.9 \mathrm{~Hz}\right), 7.06\left(2 \mathrm{H}_{\mathrm{A}}+2 \mathrm{H}_{\mathrm{B}}, \mathrm{d}, J 8.4 \mathrm{~Hz}\right), 7.24-7.33\left(2 \mathrm{H}_{\mathrm{A}}+\right.$ $\left.2 \mathrm{H}_{\mathrm{B}}, \mathrm{m}\right), 7.62-7.71\left(2 \mathrm{H}_{\mathrm{A}}+2 \mathrm{H}_{\mathrm{B}}, \mathrm{m}\right), 7.91\left(1 \mathrm{H}_{\mathrm{A}}, \mathrm{s}\right), 7.97\left(1 \mathrm{H}_{\mathrm{B}}, \mathrm{s}\right) .{ }^{13} \mathrm{C} \mathrm{NMR}\left(\mathrm{CDCl}_{3}, 75 \mathrm{MHz}\right)$ $\delta 43.65,44.07,51.66,52.47,52.57,67.01,69.18,83.24,84.26,101.34,102.04,122.53$ (q, J 273.61 Hz), 122.62 (q, J 273.61 Hz), 124.53-124.68 (2C, m), 127.30, 127.58, 129.43, 129.47, 129.60, 130.17, 131.16, 131.19, 132.63 (q, J 33.80 Hz), 132.92, 133.37 (q, J $33.98 \mathrm{~Hz}), 134.96,135.21$, $135.35,158.59,159.58,162.80,162.92$ (two carbons are accidentally isochronous). HRMS (ESI) $m / z[\mathrm{M}+\mathrm{H}]^{+} 587.0477$ (calcd for $\mathrm{C}_{22} \mathrm{H}_{18} \mathrm{ClF}_{6} \mathrm{~N}_{2} \mathrm{O}_{6} \mathrm{~S}^{+}$, 587.0473).

\subsection{Microbiology}

\subsubsection{Antibacterial Assay}

Following a previously reported procedure [31], the minimum inhibitory concentration (MIC), i.e., the lowest concentration of the tested compound, expressed as $\mu \mathrm{g} / \mathrm{mL}(\gamma)$, which prevented any visible growth of the stated bacteria, was evaluated. Briefly, MIC determinations were performed by the microdilution method, according to the Clinical laboratory Standards Institute (CLSI) guidelines with few modifications. The MIC values were obtained through duplicate experiments.

Compounds 5-26 were tested on the following microorganisms: Gram-positive bacteria: S. aureus (ATCC 29213), S. aureus (ATCC 6538 P), S. aureus (ATCC 6538), E. faecalis (ATCC 29212); Gram-negative bacteria: P. aeruginosa (ATCC 27853), E. coli (ATCC 25922), K. pneumoniae (ATCC 13883); fungi: C. albicans (ATCC 10231), C. albicans (ATCC 90028), C. glabrata (ATCC 15126), C. tropicalis (ATCC 750), C. kefyr (ATCC 204,093 C. krusei (ATCC 6258); and clinical isolates C. albicans 810, C. krusei 31A29, C. parapsilosis 11A13, C. parapsilosis 1A1 (from the Biomedical Sciences and Human Oncology Department of the University of Bari Aldo Moro, Bari, Italy).

\subsubsection{Evaluation of Membrane Permeability}

Overnight cultures of S. aureus (ATCC 6538 P) and E. faecalis (ATCC 29212) were freshly inoculated $(50 \mu \mathrm{L})$ in $5 \mathrm{~mL}$ of Mueller Hinton Broth (MHB) and incubated at $37^{\circ} \mathrm{C}$ for 240 min until reaching a final optical density at $600 \mathrm{~nm}$ of $0.2 \pm 0.3$ (corresponding to $9.47 \pm 0.14 \log \mathrm{CFU} / \mathrm{mL}$, on average). After centrifugation at $9500 \mathrm{rpm}, 4^{\circ} \mathrm{C}$ for $20 \mathrm{~min}$, the supernatant was discarded, and the pellet was washed twice with $0.01 \mathrm{M}$ potassium phosphate buffer solution (PBS, pH 7.4) before being re-suspended in the same volume of PBS. Then, $100 \mu \mathrm{L}$ of each bacterial suspension was transferred to Eppendorf tubes containing $1 \mathrm{~mL}$ of PBS supplemented with 18, 21 and 22 at concentrations equal to their corresponding MIC values or four, eight and 10-fold higher. Different concentrations of the antibiotic bacitracin $(0.1,0.4,0.8$ and $1 \mu \mathrm{g} / \mathrm{mL})$ were added as positive control. Control samples were prepared similarly without treatment. Test tubes, in triplicates, were incubated at $37{ }^{\circ} \mathrm{C}$ for $1 \mathrm{~h}$ at 10 strokes. After incubation, the cells were harvested at 12,000 rpm for $5 \mathrm{~min}$ and re-suspended in PBS $(200 \mu \mathrm{L})$ containing $10 \mu \mathrm{g} / \mathrm{mL}$ of crystal violet $(\mathrm{CV})$. The samples were incubated for $10 \mathrm{~min}$ at $37^{\circ} \mathrm{C}$. The suspension was then centrifuged at 12,000 rpm for $15 \mathrm{~min}$ and the OD of the cell-free supernatant was measured at $570 \mathrm{~nm}$. The percentage of CV uptake of all the samples was calculated using the following formula:

$$
\text { CV uptake }(\%)=((\text { OD570nm test }) /(\text { OD570nm CV solution })) \times 100
$$

\subsubsection{Statistical Analysis}

A one-way between-subjects ANOVA was conducted by using SPSS 20.0 (IBM SPSS, Armonk, NY, USA) to compare the effect of different concentrations (expressed in relation to respective MIC) of each assayed molecule on CV uptake percentage (\%) after incubation with cells from two different target strains. Homogeneity of variances was checked using Levene's test $(p<0.05)$. Multi-comparison analyses of means were performed with Tukey's HSD post hoc test $(p<0.05)$. 


\subsection{Cytotoxicity Assay}

This assay was performed following our previously reported procedure on HepG2 (Sigma-Aldrich, St. Louis, MO) cells [29]. Briefly, HepG2 cells were cultured in MEM with Earle's Salts (Euroclone) supplemented with 10\% (v/v) fatal bovine serum (Sigma-Aldrich. ST. Louis, MO), $2 \mathrm{mM}$ L-glutamine (Sigma-Aldrich, St. Louis, MO), $100 \mathrm{mg} / \mathrm{mL}$ penicillin and $100 \mathrm{mg} / \mathrm{mL}$ streptomycin (Sigma-Aldrich, St. Louis, MO) at $37^{\circ} \mathrm{C}$ in $5 \% \mathrm{CO}_{2}$. The cells, grown to $70 \%$ confluence, were trypsinized using Trypsin-EDTA 1X in PBS (Aurogene) and plated in 96-well plates at a density of 10,000 cells per well in $125 \mu \mathrm{L}$ of cell culture medium. Assays were performed $24 \mathrm{~h}$ after cells were seeded.

HepG2 cell viability was assessed using a conventional 3-(4,5-dimethylthiazol-2-yl)2,5-diphenyltetrazolium bromide (MTT) reduction assay which is based on the ability of viable cells to metabolize MTT, a water-soluble salt, by cellular oxidoreductases into a waterinsoluble blue formazan product [32]. The amount of formazan produced is correlated to the viable cells (see the cited paper for details). The cells are incubated for $24 \mathrm{~h}$ at $37^{\circ} \mathrm{C}$ in $5 \% \mathrm{CO}_{2}$ with different concentrations of test compounds, then each well is supplemented with a solution of MTT in PBS (50 ug/mL final concentration). After $3 \mathrm{~h}$ of incubation, this solution is removed and $100 \mu \mathrm{L}$ of DMSO/abs. EtOH 1:1 is added to each well. Absorbance values are measured at $570 \mathrm{~nm}$ using a Victor V3 plate reader (PerkinElmer) and DMSO medium is used as blank solution. Absorbance values are measured at $570 \mathrm{~nm}$ using a Victor V3 plate reader (PerkinElmer) and DMSO medium is used as blank solution. At least three independent experiments with six replicates $(n 18)$ were carried out, and the results were averaged. The curve obtained for the determination of the concentration of compound 22 that caused a $50 \%$ reduction in the number of HepG2 viable cells ( $\left.\mathrm{IC}_{50}\right)$ is shown in Figure 5.

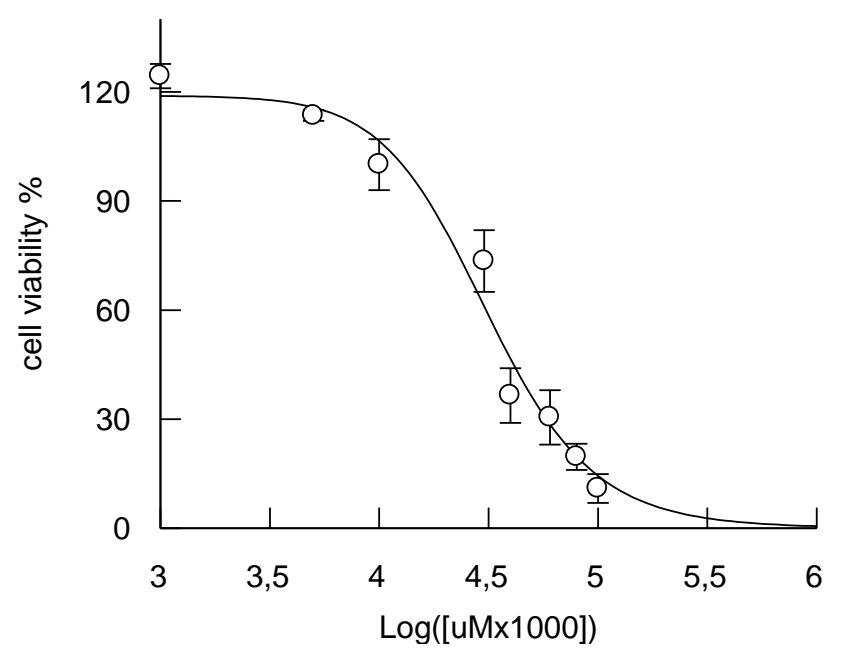

Figure 5. Determination of the concentration of compound 22 that caused a $50 \%$ reduction in the number of HepG2 viable cells ( $\left.\mathrm{IC}_{50}\right)$.

\subsection{Quantum Mechanical Calculations}

Calculations were performed according to the procedures we previously developed [23,24]. To check the reliability of the procedure, we verify if it was able to predict the cis/trans diastereomer equilibrium found in an apolar solvent $\left({ }^{1} \mathrm{H} \mathrm{NMR}, \mathrm{CDCl}_{3}\right)$. To begin with, we focused on the relatively simple congener 5 . Briefly, the model of both cis and trans diastereomers of compound $\mathbf{5}$ were generated from the atomic fragments incorporated into Spartan'16 (Wavefunction Inc., Irvine, CA, USA) inner fragment library and assuming the suggested default starting geometries. The generated geometries were optimized by the molecular mechanics MMFF routine offered by the software [33] and then submitted to a systematic conformational distribution analysis using the default step sizes. All conformers in a window of $10 \mathrm{Kcal} / \mathrm{mol}$ above the global minimum conformer were retained. When 
two conformers differed by dihedral values lower than $10^{\circ}$, the less stable conformer was left out. Conformers were then classified according to their ab initio gas-phase energy content calculated at the RHF/6-31G* level. All conformers falling within a window of $5 \mathrm{kcal} / \mathrm{mol}$ above the global minimum were retained and submitted to RHF/6-31G* geometry optimization. After removal of redundant conformers (i.e., each conformer differing from a more stable one by less than $5^{\circ}$ in their corresponding dihedral values), the so-obtained set of conformers underwent geometry optimization by density functional theory (DFT) implemented in Spartan'16 with B3LYP functional [34] and the 6-31G* basis set [35] in the gas phase. The optimized structures were confirmed as real minima by IR frequency calculation (DFT B3LYP/6-31G*//DFT B3LYP/6-31G*). The above geometry optimization was performed also applying the conductor-like polarizable continuum model (C-PCM; Spartan'16) to allow for apolar solvating effect consideration [36,37]. Based on the difference found between the energies of the so-obtained cis- and trans- 5 global minimum conformers $(1.62 \mathrm{kcal} / \mathrm{mol})$ a 17:1 cis/trans equilibrium ratio was found and this was in agreement with what observed through ${ }^{1} \mathrm{H}$ NMR analysis. The so-obtained cis-5 global minimum conformer was used to obtain the corresponding model for compound 22 whose geometry was in turn optimized by DFT implemented in Spartan'20 with $\tilde{\omega}$ B97X-D functional [38] and the 6-31G* basis set. For IR frequency calculation, the less costly EDF2 DFT model was used (basis set: 6-31G*).

\section{Conclusions}

When treating human infectious diseases caused by unknown microorganisms, wide spectrum antibiotics are obviously preferred. However, these powerful agents cause socalled 'collateral damages' (selection of resistant strains and unwanted development of colonization or infection with multidrug-resistant organisms) [39]. Furthermore, wide spectrum antimicrobials generally affect also the vitality of the microorganisms of the host microbiota. The gut microbiota is involved in the regulation of numerous physiological processes including appetite and metabolic status [40]. It has been demonstrated that an imbalance of pathogens and beneficial bacteria may have deleterious effects on the host. [41]. For example, alterations in the gut microbiota may lead to hepatocellular carcinoma [42]. Thus, when the causative pathogen is diagnosed, selective antibacterial agents should be preferred to face the infection.

Some of the nitroazetidines reported herein performed as selective and potent antibacterial agents on Gram-positive bacteria (E. faecalis and three strains of S. aureus), with the most potent congener being the $p$-chlorobenzyl, $p$-tolyl decorated nitroazetidine $\mathbf{2 2}$ which displayed potency close to that of norfloxacin, the reference antibiotic (minimum inhibitory concentration values 4 and $1-2 \mu \mathrm{g} / \mathrm{mL}$, respectively). Since 22 is a relatively efficient agent against Gram-positive bacteria and displayed cytotoxicity on eucharyotic cells only at 4-times higher concentrations (inhibiting concentration on 50\% of the cultured eukaryotic cells: $36 \pm 10 \mu \mathrm{M}$ ), this nitroazetidine may be considered as a promising hit compound for the development of a new series of antibacterials selectively active on Gram-positive pathogens. The relatively concise synthetic route described herein, based as it is on easily available starting materials, could feed further structure-activity relationship studies, thus allowing for the fine investigation and optimization of the toxico-pharmacological profile.

Author Contributions: Conceptualization, G.L., C.T. and G.P.; resources, G.P., G.L., A.R. and L.C.; writing—original draft preparation, G.L., C.T., G.P. and L.Q.; writing—review and editing, C.T. and M.M.C.; investigation, G.L., C.T., A.B., L.B. and L.S.; methodology, A.D.P., L.Q. and L.S.; supervision, A.R., L.C., G.L. and G.P. All authors have read and agreed to the published version of the manuscript.

Funding: Financial support was provided by grants by Genoa University (FRA 2017).

Institutional Review Board Statement: Not applicable.

Informed Consent Statement: Not applicable.

Data Availability Statement: Not applicable. 
Acknowledgments: G.L. and A.D.P. are indebted to Alexia Barbarossa for her technical help and suggestions.

Conflicts of Interest: The authors declare no conflict of interest.

Sample Availability: Samples of the compounds are available from the authors.

\section{References}

1. Levy, S.B. The Antibiotic Paradox: How Miracle Drugs Are Destroying the Miracle; Plenum Publishers: New York, NY, USA, 1992.

2. Navidinia, M. The clinical importance of emerging ESKAPE pathogens in nosocomial infections. J. Paramed. Sci. 2016, 7, 2008-4978.

3. Tacconelli, E.; Carrara, E.; Savoldi, A.; Harbarth, S.; Mendelson, M.; Monnet, D.L.; Pulcini, C.; Kahlmeter, G.; Kluytmans, J.; Carmeli, Y.; et al. Discovery, research, and development of new antibiotics: The WHO priority list of antibiotic-resistant bacteria and tuberculosis. Lancet Infect. Dis. 2018, 18, 318-327. [CrossRef]

4. Mulani, M.S.; Kamble, E.E.; Kumkar, S.N.; Tawre, M.S.; Pardesi, K.R. Emerging Strategies to Combat ESKAPE Pathogens in the Era of Antimicrobial Resistance: A Review. Front. Microbiol. 2019, 10, 539. [CrossRef] [PubMed]

5. SciFindern Home Page. Available online: https://scifinder-n.cas.org (accessed on 13 April 2021).

6. Parkes, A.L. Antibacterial medicinal chemistry-what can we design for? Expert Opin. Drug Discov. 2020, 15, 1005-1013. [CrossRef]

7. Singh, S.B.; Young, K.; Miesel, L. Screening strategies for discovery of antibacterial natural products. Expert Rev. Anti-Infe. 2011, 9, 589-613.

8. Lazzara, P.R.; Moore, T.W. Scaffold-hopping as a strategy to address metabolic liabilities of aromatic compounds. RSC Med. Chem. 2020, 11, 18-29. [CrossRef] [PubMed]

9. Fuson, R.C. The Principle of Vinylogy. Chem. Rev. 1935, 16, 1-27. [CrossRef]

10. Sharma, R.; Samadhiya, P.; Srivastava, S.D.; Srivastava, S.K. Synthesis and biological activity of 2-oxoazetidine derivatives of phenothiazine. Org Commun. 2011, 4, 42-51.

11. Patel, R.; Bhandari, A. Synthesis and Antimicrobial Screening of Some Azetidine Derivatives. Am. J. Adv. Drug Deliv. 2014, 2, 104-109.

12. Tavani, C.; Bianchi, L.; Giorgi, G.; Maccagno, M.; Petrillo, G. Densely Functionalized 2-Methylideneazetidines from Nitrodienic Building Blocks. Eur. J. Org. Chem. 2018, 126-136. [CrossRef]

13. Sana, S.; Datta, S.; Biswas, D.; Sengupta, D. Assessment of synergistic antibacterial activity of combined biosurfactants revealed by bacterial cell envelop damage. BBA Biomembr. 2018, 1860, 579-585. [CrossRef] [PubMed]

14. Li, N.; Luo, M.; Fu, Y.J.; Zu, Y.G.; Wang, W.; Zhang, L.; Yao, L.P.; Zhao, C.J.; Sun, Y. Effect of corilagin on membrane permeability of Escherichia coli, Staphylococcus aureus and Candida albicans. Phytother. Res. 2013, 27, 1517-1523. [CrossRef] [PubMed]

15. Da Cruz Nizer, W.S.; Ferraz, A.C.; Moraes, T.D.F.S.; Lima, W.G.; Dos Santos, J.P.; Duarte, L.P.; Ferreira, J.M.S.; de Brito Magalhães, C.L.; Vieira-Filho, S.A.; Andrade, A.C.S.P.; et al. Pristimerin isolated from Salacia crassifolia (Mart. Ex. Schult.) G. Don. (Celastraceae) roots as a potential antibacterial agent against Staphylococcus aureus. J. Ethnopharmacol. 2021, 266, 113423. [CrossRef]

16. Shai, Y. Mechanism of the binding, insertion and destabilization of phospholipid bilayer membranes by $\alpha$-helical antimicrobial and cell non-selective membrane-lytic peptides. BBA Biomembr. 1999, 1462, 55-70. [CrossRef]

17. The Bacterial Diversity Metadatabase. Available online: https://bacdive.dsmz.de/ (accessed on 11 June 2021).

18. Revell, K.D.; Heldreth, B.; Long, T.E.; Jang, S.; Turos, E. N-thiolated $\beta$-lactams: Studies on the mode of action and identification of a primary cellular target in Staphylococcus aureus. Bioorg. Med. Chem. 2007, 15, 2453-2467. [CrossRef]

19. Lanne, A.; Cui, Y.; Browne, E.; Craven, P.G.E.; Cundy, N.J.; Coltman, N.J.; Dale, K.; Feula, A.; Frampton, J.; Goff, A. Azetidines kill Mycobacterium tuberculosis without detectable resistance by blocking mycolate assembly. bioRxiv 2020. [CrossRef]

20. Boyd, D.B. $\beta$-Lactam Antibacterial Agents: Computational Chemistry Investigations. In The Amide Linkage; Greenberg, A., Breneman, C.M., Liebman, J.F., Eds.; Wiley: New York, NY, USA, 2000; pp. 337-375.

21. Cooper, R.D.G.; De Marco, P.V.; Cheng, J.C.; Jones, N.D. Structural Studies on Penicillin Derivatives. J. Am. Chem. Soc. 1969, 91, 1408-1415. [CrossRef] [PubMed]

22. Dexter, D.D.; van der Veen, J.M. Conformations of penicillin G: Crystal structure of procaine penicillin G monohydrate and a refinement of the structure of potassium penicillin G. J. Chem. Soc. Perkin I. 1978, 185-190. [CrossRef]

23. Carocci, A.; Roselli, M.; Budriesi, R.; Micucci, M.; Desaphy, J.F.; Altamura, C.; Cavalluzzi, M.M.; Toma, M.; Passeri, G.I.; Milani, G. Synthesis and Evaluation of Voltage-Gated Sodium Channel Blocking Pyrroline Derivatives Endowed with Both Antiarrhythmic and Antioxidant Activities. Chem. Med. Chem. 2021, 16, 578-588. [CrossRef]

24. Gnocchi, D.; Cavalluzzi, M.M.; Mangiatordi, G.F.; Rizzi, R.; Tortorella, C.; Spennacchio, M.; L28ntini, G.; Altomare, A.; Sabbà, C.; Mazzocca, A. Xanthenylacetic Acid Derivatives Effectively Target Lysophosphatidic Acid Receptor 6 to Inhibit Hepatocellular Carcinoma Cell Growth. Chem. Med. Chem. 2021, 16, 1-10.

25. Smith, D.J.; Anderson, R.C. Toxicity and Metabolism of Nitroalkanes and Substituted Nitroalkanes. Agric. Food Chem. 2013, 61, 763-779. [CrossRef]

26. Cavalluzzi, M.M.; Mangiatordi, G.F.; Nicolotti, O.; Lentini, G. Ligand Efficiency Metrics in Drug Discovery: The Pros and Cons from a Practical Perspective. Expert Opin. Drug Discov. 2017, 12, 1087-1104. [CrossRef] [PubMed] 
27. Roselli, M.; Carocci, A.; Budriesi, R.; Micucci, M.; Toma, M.; Di Cesare Mannelli, L.; Lovece, A.; Catalano, A.; Cavalluzzi, M.M.; Bruno, C.; et al. Synthesis, Antiarrhythmic Activity, and Toxicological Evaluation of Mexiletine Analogues. Eur. J. Med. Chem. 2016, 121, 300-307. [CrossRef] [PubMed]

28. Ghose, A.K.; Crippen, G.M. Atomic Physicochemical Parameters for Three-Dimensional-Structure-Directed Quantitative Structure-Activity Relationships. 2. Modeling Dispersive and Hydrophobic Interactions. J. Chem. Inf. Comp. Sci. 1987, 27, 21-35. [CrossRef] [PubMed]

29. Chattaraj, P.K.; Sarkar, U.; Roy, D.R. Electrophilicity Index. Chem. Rev. 2006, 106, 2065-2091. [CrossRef] [PubMed]

30. Benzi, A.; Bianchi, L.; Giorgi, G.; Maccagno, M.; Petrillo, G.; Tavani, C. 2-Aryl-3-Vinyl Substituted Imidazo [1,2-a] pyridines and Fluorescent Electrocyclization Derivatives therefrom. ChemistrySelect 2020, 5, 4552-4558. [CrossRef]

31. Cavalluzzi, M.M.; Budriesi, R.; De Salvia, M.A.; Quintieri, L.; Piarulli, M.; Milani, G.; Gualdani, R.; Micucci, M.; Corazza, I.; Rosato, A.; et al. Lubeluzole: From Anti-Ischemic Drug to Preclinical Antidiarrheal Studies. Pharmacol. Rep. 2021, 73, 172-184. [CrossRef]

32. Berridge, M.V.; Tan, A.S. Characterization of the cellular reduction of 3-(4,5-dimethylthiazol-2-yl)-2,5-diphenyltetrazolium bromide (MTT): Subcellular localization, substrate dependence, and involvement of mitochondrial electron transport in MTT reduction. Arch. Biochem. Biophys. 1993, 303, 474-482. [CrossRef]

33. Halgren, T.A. Merck Molecular Force Field. I.; Basis, Form, Scope, Parameterization, and Performance of Mmff94. J. Comput. Chem. 1996, 17, 520-552. [CrossRef]

34. Becke, A.D. Density-Functional Exchange-Energy Approximation with Correct Asymptotic Behavior. Phys. Rev. A 1988, 38, 3098-3100. [CrossRef]

35. Davidson, E.R.; Feller, D. Basis Set Selection for Molecular Calculations. Chem. Rev. 1986, 86, 681-696. [CrossRef]

36. Lange, A.W.; Herbert, J.M.A. Smooth, Nonsingular, and Faithful Discretization Scheme for Polarizable Continuum Models: The Switching/Gaussian Approach. J. Chem. Phys. 2010, 133, 244111. [CrossRef] [PubMed]

37. Lange, A.W.; Herbert, J.M. Symmetric versus Asymmetric Discretization of the Integral Equations in Polarizable Continuum Solvation Models. Chem. Phys. Lett. 2011, 509, 77-87. [CrossRef]

38. Chai, J.-D.; Head-Gordon, M. Long-Range Corrected Hybrid Density Functionals with Damped Atom-Atom Dispersion Corrections. Phys. Chem. Chem. Phys. 2008, 10, 6615-6620. [CrossRef] [PubMed]

39. Paterson, D.L. "Collateral Damage" from Cephalosporin or Quinolone Antibiotic Therapy. Clin. Infect. Dis. 2004, 38, 341-345. [CrossRef] [PubMed]

40. Wijdeveld, M.; Nieuwdorp, M.; IJzerman, R. The Interaction between Microbiome and Host Central Nervous System: The Gut-Brain Axis as a Potential New Therapeutic Target in the Treatment of Obesity and Cardiometabolic Disease. Expert Opin. Ther. Tar. 2020, 24, 639-653. [CrossRef] [PubMed]

41. Hartmann, P.; Chu, H.; Duan, Y.; Schnabl, B. Gut Microbiota in Liver Disease: Too Much Is Harmful, Nothing at All Is Not Helpful Either. Am. J. Physiol. Gastrointest. Liver Physiol. 2019, 316, G563-G573. [CrossRef]

42. Zhou, A.; Tang, L.; Zeng, S.; Lei, Y.; Yang, S.; Tang, B. Gut Microbiota: A New Piece in Understanding Hepatocarcinogenesis. Cancer Lett. 2020, 474, 15-22. [CrossRef] 